\title{
Dynamic Characteristics and Load Sharing of Herringbone Wind Power Gearbox
}

\author{
Shuai Mo $\mathbb{D}^{1,2,3}$ Ting Zhang $\mathbb{D}^{1,2}$ Guoguang Jin $\mathbb{D}^{1,2}$, Zhanyong Feng $\mathbb{D}^{1,2}$ \\ Jiabei Gong $\mathbb{D},{ }^{1,2}$ and Shengping Zhu $\mathbb{D}^{1,2}$ \\ ${ }^{1}$ School of Mechanical Engineering, Tianjin Polytechnic University, Tianjin 300387, China \\ ${ }^{2}$ Tianjin Key Laboratory of Modern Electromechanical Equipment Technology, Tianjin 300387, China \\ ${ }^{3}$ The State Key Laboratory of Materials Processing and Die \& Mould Technology, Huazhong University of Science \& Technology, \\ Wuhan 430074, China \\ Correspondence should be addressed to Guoguang Jin; jinguoguang@tjpu.edu.cn
}

Received 21 May 2018; Accepted 18 October 2018; Published 31 October 2018

Academic Editor: Jaime Gallardo-Alvarado

Copyright (C) 2018 Shuai Mo et al. This is an open access article distributed under the Creative Commons Attribution License, which permits unrestricted use, distribution, and reproduction in any medium, provided the original work is properly cited.

In this study, the dynamic model for the herringbone planetary gear transmission system is established by the lumped parameter method based on the system dynamics and the Lagrange equation, and the impact of the support stiffness and the torsional stiffness on dynamic characteristics is studied. The research results have a guiding significance for the design of the herringbone gear transmission system. In this model, the herringbone gear is treated as a special gear coupled by 2 opposite helical gears, where the stagger angle, comprehensive meshing error, support stiffness, support damping, and load inertia are considered in the analysis of dynamics. Moreover, the dynamic characteristic of the carrier is considered as well. By calculating the meshing force curve of the transmission system, the impact of the stagger angle, supporting stiffness, and the torsional stiffness on meshing force and load sharing coefficient is analyzed. The results show that the stagger angle has an obvious impact on load sharing coefficient while it has little impact on maximum meshing force. And the support stiffness has a more obvious impact on the dynamic characteristics of the system. The recommendary support stiffness of the system is that all of the support stiffness of the sun gear, planetary gear, ring gear, and carrier is $10^{7} \mathrm{~N} / \mathrm{m}$. The torsional stiffness has little impact on the dynamic characteristics of transmission system, except the torsional stiffness of planetary gear, and carrier has an obvious impact on load sharing coefficient. The commercial software ADAMS carried out dynamics analysis of the transmission system to verify the necessity validity of the theoretical analysis.

\section{Introduction}

Herringbone gear planetary transmission system, which is one of the most important parts of wind power generator, is wildly used in ship, aviation, and other transmission fields because of its distinctive advantages, such as small volume, light weight, large transmission ratio, strong bearing capacity, high transmission efficiency, and so on. The load distribution of planetary gears in the transmission directly affects the working life, stability, and reliability of gears. It is of great practical and engineering significance to carry out the dynamic load characteristics of herringbone gear transmission system.

Many scholars around the world have done many theoretical and experimental studies on the load sharing characteristics of planetary transmission. SHI Wan-kai et al. [1] studied the transmission dynamic characteristic of wind speed increase gearbox to study the effect on wind turbine gearbox dynamic load characteristics caused by stagger angle and left-right coupling torsional stiffness. ZHANG Lin-lin et al. [2] researched load sharing characteristic of herringbone gear transmission system influenced by meshing phase and conducted an experiment on different meshing phase planetary transmission system to study load sharing on single-stage herringbone planetary gear test stand. MO Shuai et al. [35] proposed a new dynamic model of herringbone star gear transmission system to study meshing error, tooth thickness error, base circle error, and assembly error influencing the load sharing characteristic of GTF aeroengine. Ming LI [6] established a model to predict the reliability of helicopter 
planetary gear train under the condition with partial load and analyzed structure and part load state of the transmission system in detail based on the study of load sharing characteristics of a helicopter gearbox. The impact of assembly error on load sharing characteristic of closed differential herringbone gear transmission system was researched by ZHU Zeng-bao [7]. LU Jun-hua [8] established a calculation model of $2 \mathrm{~K}$ $\mathrm{H}$ transmission system to analyze load sharing characteristic of planetary transmission system from the point of view of dynamics. XU Xiang-yang [9] studied impact of stiffness of flexible pin shaft on load sharing of star transmission system and verified the effectiveness of theoretical result. Kahraman A et al. [10-14] researched the impact of gear indexing error on dynamic response of a typical multigrid gear system and compared subharmonic resonance of response with numerical integration result directly to prove the accuracy of prediction and predicted other nonlinear phenomena by nonlinear model and studied the variation of the position errors of the planetary gear and the accompanying star wheel under the condition of light load in the small module planetary gear system as well. Howard I [15] proposed a new dynamic model of flexible supporting gear train to calculate the meshing stiffness with varied gear center distance and coupled it to dynamic model by iteration. Wang J et al. [16] developed a three free degrees torsional-vibration model of locomotive spur gear transmission system, where nonlinear gap, static transfer error, and time varying meshing stiffness and other factors were considered, and the influence of speed and stiffness of pinion on the dynamic characteristics of the transmission system is studied by numerical method. Fernandez-Del-Rincon A et al. [17] proposed a model which can simultaneously consider the internal excitation caused by variable meshing stiffness and the variable compliance results of bearing. The model combines with hybrid formula for calculating meshing force and nonlinear variable flexibility of bearing to prove a detail described for meshing force. Liang $\mathrm{X}$ et al. [18] focus on modeling, detection, and diagnosis of gearbox failure based on dynamics. The research result was limited on some key and basic aspects by literature review: gear meshing stiffness evaluation, gearbox damage modeling and fault diagnosis technology, gearbox transmission path modeling, and method verification. TAN Yuan-qiang [19] researched dynamic and load sharing characteristics of closed differential planetary gearbox by comparative trial, which proves that the average load performance of the closed stage is better than that of the differential stage, and the greater the load, the better the load performance of the two stages. The transmission performance of the planetary system with the three kinds of load sharing methods is better than the single load sharing system. Singh [20] established an analysis model of planetary transmission load sharing characteristic to physically explain basic mechanism of the phenomenon which causes inhomogeneous load. Wink et al. [21] researched the calculation method of load distribution of tooth surface and proved three different methods to solve load distribution of gear teeth in calculation of static transmission error of gear pair.

The herringbone gear planetary transmission system of wind power train was taken as the research object in this paper. The support stiffness, support damping, meshing stiffness, meshing damping, and other factors of herringbone gear in the condition of stagger angle which is 0 are overall considered to study their impact on dynamic characteristic. Compared with other papers, this paper especially researched the impact of carrier on dynamic characteristic and deduced the formula of carrier as one part of system dynamics formula. The dynamic model of herringbone gear planetary transmission system is established to analyze the dynamic characteristics of the system. The theoretical dynamic characteristic of system is verified through simulation.

\section{Dynamics Theories and Equations}

2.1. Dynamic Model of Planetary Transmission System. The three-dimensional model of herringbone planetary transmission system is shown in Figure 1.

The impact of the input and output shafts is not considered in this paper. Thus, the input torque is directly acting on carrier. Because each single-helical gear, respectively, has the translational motion along the direction of $x, y$, and $z$ axes and turning motion in the direction of the $z$ axis, each herringbone gear, which is coupled by 2 helical gear with opposite direction, has 8 free degrees. The carrier has the translational motion along the direction of $x, y$, and $z$ axes and turning motion in the direction of the $z$ axis. Thus, the carrier has 4 free degrees. Because the herringbone gear is divided into left and right parts, subscript L means the left side gear while subscript $\mathrm{R}$ means the right-side gear. The transmission system has $X=8 N+20$ free degrees, and the generalized coordinate is

$$
X=\left\{\begin{array}{c}
x_{s L}, y_{s L}, z_{s L}, \theta_{s L}, x_{s R}, y_{s R}, z_{s R}, \theta_{s R} \\
x_{p i L}, y_{p i L}, z_{p i L}, \theta_{p i L}, x_{p i R}, y_{p i R}, z_{p i R}, \theta_{p i R} \\
x_{r L}, y_{r L}, z_{r L}, \theta_{r L}, x_{r R}, y_{r R}, z_{r R}, \theta_{r R} \\
x_{c}, y_{c}, z_{c}, \theta_{c}
\end{array}\right\}
$$

where $x, y, z$, respectively, are lateral, longitudinal, and axial displacement of a component and $\theta$ is the angle displacement of the component. $i=1,2, \ldots, N$ is the $i$ th planetary gear.

According to the $3 \mathrm{D}$ model of herringbone planetary transmission system, the dynamic model of herringbone planetary transmission system is established, as shown in Figure 2.

$O X Y$ is the fixed coordinate system, and $o x y$ is the moving reference system.

To simplify the angle displacement as the equivalent line displacement on the corresponding base circle,

$$
\begin{gathered}
u_{s i}=r_{b s} \times \theta_{s i} \\
u_{r i}=r_{b r} \times \theta_{r i} \\
u_{c}=r_{c} \times \theta_{c} \\
u_{p i j}=r_{b p j} \times \theta_{p i j}
\end{gathered}
$$




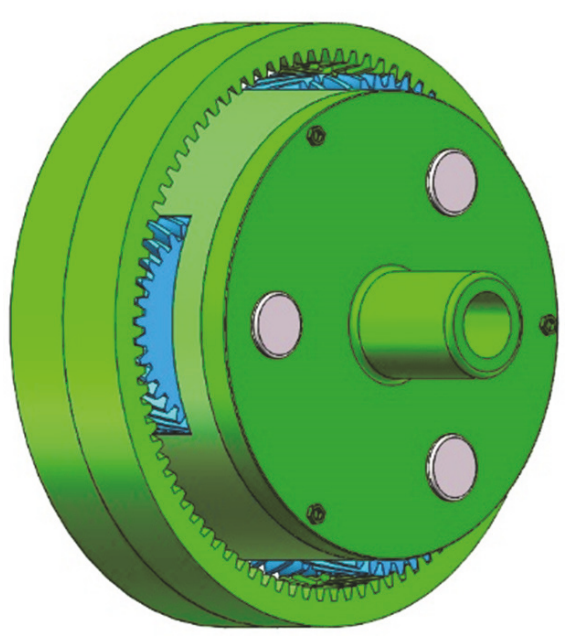

(a) 3D model with carrier of system

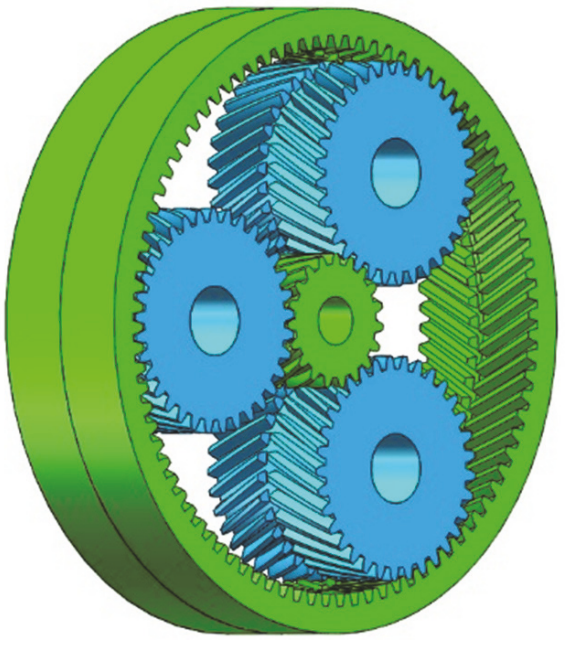

(b) 3D model without carrier of system

FIGURE 1: Three-dimensional model of herringbone planetary transmission system.

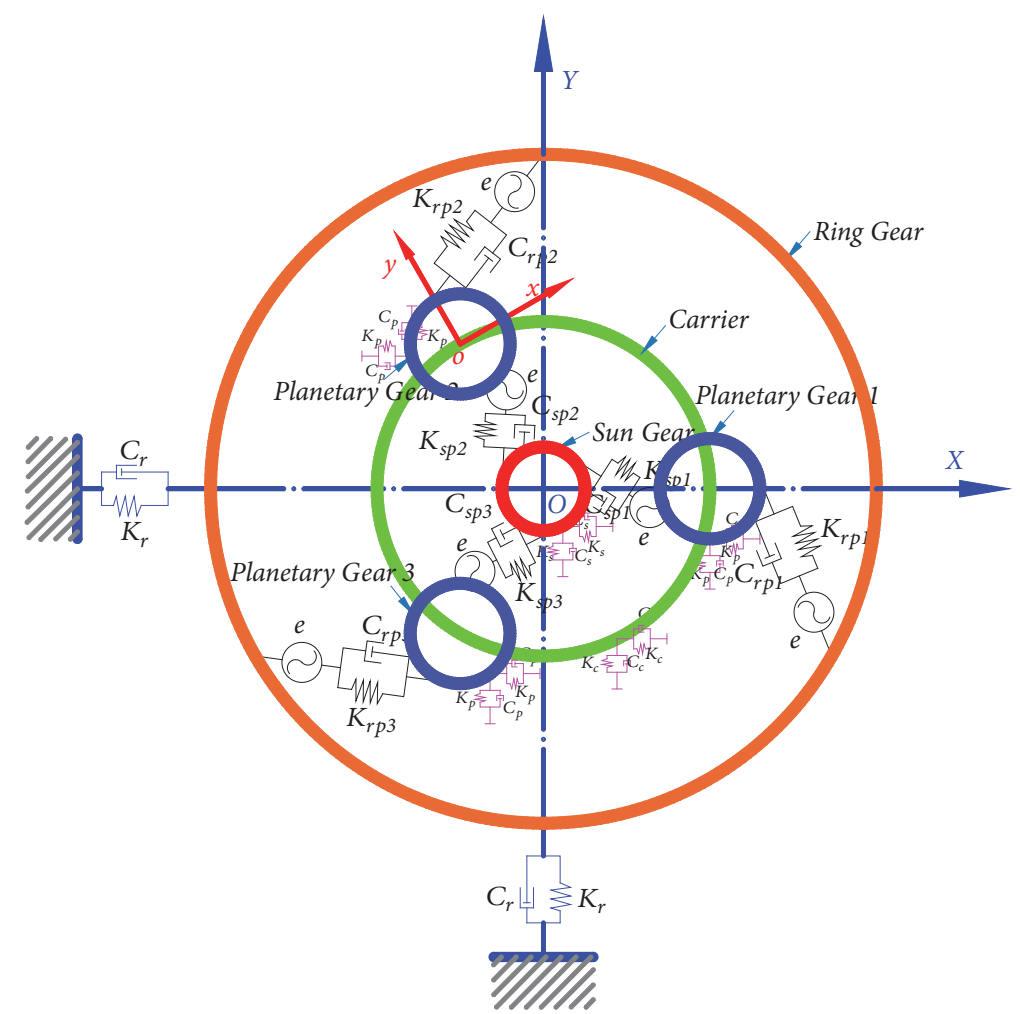

FIGURE 2: Dynamics model of herringbone planetary transmission system.

where $i=L, R$ means the left side and right side of the component. $j=1,2, \ldots, N$ means the $j$ th planetary gear. $r_{b s}, r_{b p j}, r_{b r}$, respectively, are the radius of base circle of sun gear, the $j$ th planetary gear, and ring gear. $r_{c}$ is the distance from the center of planetary gear to gyration center of carrier,

$$
r_{c}=r_{s}+r_{p}
$$

where $r_{s}, r_{p}$, respectively, are the radius of pitch circle of sun gear and planetary gear.

2.2. Equivalent Displacement. Figure 3 shows the projection relation of each component of the planetary gear system on the gear face.

According to Figure 3, projection of relative displacement of each component in the meshing line or coordinate system 


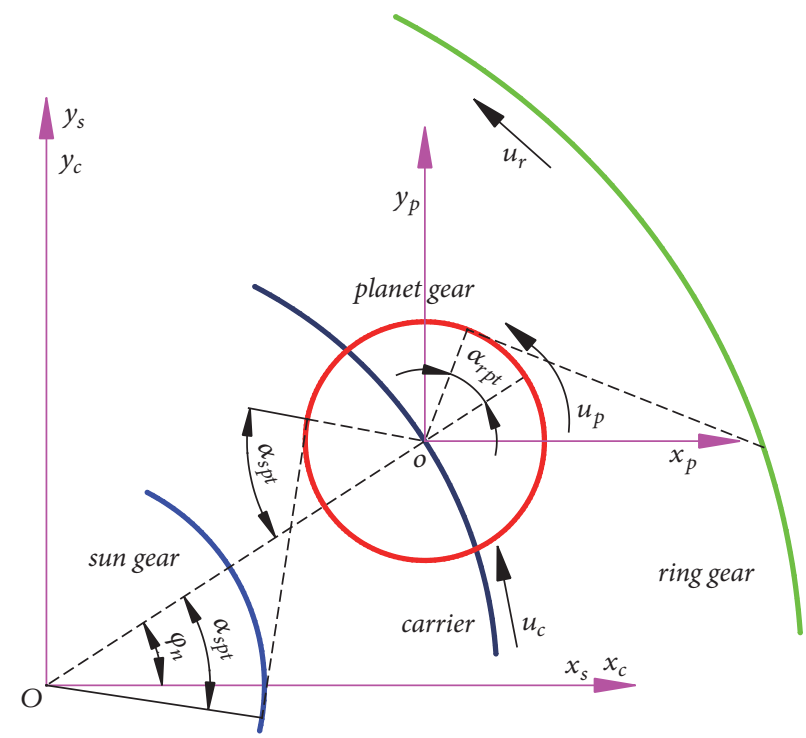

FIGURE 3: Projection relation of each component.

can be calculated. The projection of relative displacement of sun gear and planetary gear along the direction of meshing line is

$$
\begin{aligned}
& \delta_{s p i L}=\left[x_{s L} \sin \left(\phi_{p i}-\alpha_{s p i}\right)-x_{p i L} \sin \alpha_{s p}\right. \\
& +y_{s L} \cos \left(\phi_{p i}-\alpha_{s p i}\right)-y_{p i L} \cos \alpha_{s p}+r_{s} \theta_{z s L} \\
& \left.\quad+r_{p} \theta_{z p i L}\right] \cos \beta_{b}+\left(-z_{s L}+z_{p i L}\right) \sin \beta_{b}+e_{s p i}^{(L)}(t) \\
& \delta_{s p i R}=\left[x_{s R} \sin \left(\phi_{p i}-\alpha_{s p i}\right)-x_{p i R} \sin \alpha_{s p}\right. \\
& \quad+y_{s R} \cos \left(\phi_{p i}-\alpha_{s p i}\right)-y_{p i R} \cos \alpha_{s p}+r_{s} \theta_{z s R} \\
& \left.\quad+r p \theta_{z p i R}\right] \cos \beta_{b}+\left(z_{s R}-z_{p i R}\right) \sin \beta_{b}+e_{s p i}^{(R)}(t) .
\end{aligned}
$$

The projection of relative displacement of planetary gear and ring gear along the direction of meshing line is

$$
\begin{aligned}
& \delta_{r p i L}=\left[-x_{r L} \sin \left(\phi_{p i}+\alpha_{p r i}\right)-x_{p i L} \sin \alpha_{r p}\right. \\
& +y_{r L} \cos \left(\phi_{p i}+\alpha_{p r i}\right)-y_{p i L} \cos \alpha_{r p}+r_{r} \theta_{z r L} \\
& \left.+r_{r} \theta_{z p i L}\right] \cos \beta_{b}+\left(z_{r L}-z_{p i L}\right) \sin \beta_{b}+e_{r p i}^{(L)}(t) \\
& \delta_{r p i R}=\left[-x_{r R} \sin \left(\phi_{p i}+\alpha_{p r i}\right)+x_{p i R} \sin \alpha_{r p}\right. \\
& \quad+y_{r R} \cos \left(\phi_{p i}+\alpha_{p r i}\right)-y_{p i R} \cos \alpha_{r p}+r_{r} \theta_{z r R} \\
& \left.+r_{p} \theta_{z p i 2}\right] \cos \beta_{b}+\left(-z_{r R}+z_{p i R}\right) \sin \beta_{b}+e_{r p i}^{(R)}(t) .
\end{aligned}
$$

And the projection of relative displacement of planetary gear and carrier along the directions of $x_{c}, y_{c}, z_{c}$, and $u_{c}$ is

$$
\begin{gathered}
\delta_{p c x i}=x_{c}-x_{p i}-u_{c} \sin \phi_{p i} \\
\delta_{p c y i}=y_{c}-y_{p i}-u_{c} \cos \phi_{p i}
\end{gathered}
$$

$$
\begin{aligned}
& \delta_{p c z i}=z_{c}-z_{p i} \\
& \delta_{p c u i}=\left(x_{p i}-x_{c}\right) \sin \phi_{p i}+\left(y_{c}-y_{p i}\right) \cos \phi_{p i}+u_{c},
\end{aligned}
$$

where $\Phi_{p i}$ is the position angle formed by the $i$ th planetary gear relative to the first planetary gear, $\Phi_{p i}=2 \pi(n-1) / N, \alpha_{s p}$ is the end face meshing angle of sun-planetary gear meshing, and $\alpha_{r p}$ is the end face meshing angle of planetary-ring gear meshing. $e_{s p}$ is the comprehensive meshing error in the sun-planetary gear meshing line, $e_{r p}$ is the comprehensive meshing error in the planetary-ring gear meshing line, $L$ is the left side, and $R$ is the right side.

2.3. Equilibrium Differential Equation of System Dynamics. According to the Newton second law and system dynamics, the differential equation of the dynamics of sun gear can be written as (6) and (7). Equation (6) is the dynamics equation of the left side of sun gear and (7) is the dynamics equation of the right side of sun gear.

$$
\begin{aligned}
& m_{s}\left(\ddot{x}_{s L}-2 \omega_{c} \dot{y}_{s L}-\omega_{c}^{2} x_{s L}\right) \\
& \quad+\sum_{i=1}^{N}\left(K_{s p i} \delta_{s p i L}+C_{s p i} \dot{\delta}_{s p i L}\right) \sin \left(\phi_{s}-\alpha_{s p i}\right) \cos \beta_{b} \\
& \quad+K_{s} x_{s L}+C_{s} \dot{x}_{s L}=0 \\
& m_{s}\left(\ddot{y}_{s L}+2 \omega_{c} \dot{x}_{s L}-\omega_{c}^{2} y_{s L}\right) \\
& \quad+\sum_{i=1}^{N}\left(K_{s p i} \delta_{s p i L}+C_{s p i} \dot{\delta}_{s p i L}\right) \cos \left(\phi_{s}-\alpha_{s p i}\right) \cos \beta_{b} \\
& \quad+K_{s} y_{s L}+C_{s} \dot{y}_{s L}=0 \\
& m_{s} \ddot{z}_{s L}-\sum_{i=1}^{N}\left(K_{s p i} \delta_{s p i L}+C_{s p i} \dot{\delta}_{s p i L}\right) \sin \beta_{b}+K_{s a} z_{s L} \\
& \quad+C_{s a} \dot{z}_{s L}+K_{s L R}\left(z_{s L}-z_{s R}\right)+C_{s L R}\left(\dot{z}_{s L}-\dot{z}_{s R}\right)=0
\end{aligned}
$$




$$
\begin{aligned}
& \frac{I_{s}}{r_{s}} \ddot{u}_{z s L}+\sum_{i=1}^{N}\left(K_{s p i} \delta_{s p i L}+C_{s p i} \dot{\delta}_{s p i L}\right) r_{s} \cos \beta_{b} \\
& \quad+K_{1 t}\left(u_{s L}-u_{s R}\right)+C_{1 t}\left(\theta_{s L}-\theta_{s R}\right)=\frac{T_{i n}}{2} \\
& m_{s}\left(\ddot{x}_{s R}-2 \omega_{c} \dot{y}_{s R}-\omega_{c}^{2} x_{s R}\right) \\
& \quad+\sum_{i=1}^{N}\left(K_{s p i} \delta_{s p i R}+C_{s p i} \dot{\delta}_{s p i R}\right) \sin \left(\phi_{s}-\alpha_{s p i}\right) \cos \beta_{b} \\
& \quad+K_{s} x_{s R}+C_{s} \dot{x}_{s R}=0 \\
& m_{s}\left(\ddot{y}_{s R}+2 \omega_{c} \dot{x}_{s R}-\omega_{c}^{2} y_{s R}\right) \\
& \quad+\sum_{i=1}^{N}\left(K_{s p i} \delta_{s p i R}+C_{s p i} \dot{\delta}_{s p i R}\right) \cos \left(\phi_{s}-\alpha_{s p i}\right) \cos \beta_{b} \\
& \quad+K_{s} y_{s R}+C_{s} \dot{y}_{s R}=0 \\
& \quad+\ddot{z}_{s R}+\sum_{i=1}^{N}\left(K_{s p i} \delta_{s p i R}+C_{s p i} \dot{\delta}_{s p i R}\right) \sin \beta_{b}+K_{s a} z_{s R} \\
& \quad+C_{s a} \dot{z}_{s R}+K_{s L R}\left(z_{s R}-z_{s L}\right)+C_{s L R}\left(\dot{z}_{s R}-\dot{z}_{s L}\right)=0 \\
& \frac{I_{s}}{r_{s}} \ddot{u}_{z s R}+\sum_{i=1}^{N}\left(K_{s p i} \delta_{s p i R}+C_{s p i} \dot{\delta}_{s p i R}\right) r_{s} \cos \beta_{b} \\
& \left.+u_{s R}-u_{s L}\right)+C_{1 t}\left(\theta_{s R}-\theta_{s L}\right)=\frac{T_{i n}}{2} \\
& \quad
\end{aligned}
$$

where $m_{s}$ is the equivalent mass of sun gear, $K_{s}$ is the radial support stiffness of sun gear, $C_{s}$ is the radial support damping of sun gear, $K_{s a}$ is the axial support stiffness, $C_{s a}$ is the axial support damping, $K_{s 12}$ is the axial stiffness between two helical gears, $C_{s 12}$ is the axial damping between two helical gears, $K_{1 t}$ is the left-right coupling torsional stiffness, $C_{1 t}$ is the left-right coupling torsional damping, and $T_{i n}$ is the input torque.

The axial support stiffness and support damping of sun gear are as follows:

$$
\begin{aligned}
& K_{s a}=2 K_{s} \sin \beta \\
& C_{s a}=2 C_{s} \sin \beta .
\end{aligned}
$$

The differential equation of the dynamics of planetary gear can be written as (10) and (11). Equation (10) is the dynamics equation of the left side of planetary gear and (11) is the dynamics equation of the right side of planetary gear.

$$
\begin{aligned}
& m_{p}\left(\ddot{x}_{p i L}-2 \omega_{c} \dot{y}_{p i L}-\omega_{c}^{2} x_{p i L}\right) \\
& \quad-\left(K_{s p i} \delta_{s p i L}+C_{s p i} \dot{\delta}_{s p i L}\right) \sin \alpha_{s n} \cos \beta_{b} \\
& \quad+\left(K_{r p i} \delta_{r p i L}+C_{r p i} \dot{\delta}_{r p i L}\right) \sin \alpha_{r n} \cos \beta_{b}+K_{p} x_{p i L} \\
& \quad+C_{p} \dot{x}_{p i L}=0
\end{aligned}
$$

$$
\begin{aligned}
& m_{p}\left(\ddot{y}_{p i L}+2 \omega_{c} \dot{x}_{p i L}-\omega_{c}^{2} y_{p i L}\right) \\
& -\left(K_{s p i} \delta_{s p i L}+C_{s p i} \dot{\delta}_{s p i L}\right) \sin \alpha_{s n} \cos \beta_{b} \\
& \text { - }\left(K_{r p i} \delta_{r p i L}+C_{r p i} \dot{\delta}_{r p i L}\right) \cos \alpha_{r n} \cos \beta_{b} \\
& +K_{p}\left(y_{p i L}-r_{c} u_{c}\right)+C_{p}\left(\dot{y}_{p i L}-r_{c} \dot{u}_{c}\right)=0 \\
& m_{p} \ddot{z}_{p i L}+\left(K_{s p i} \delta_{s p i L}+C_{s p i} \dot{\delta}_{s p i L}\right) \sin \beta_{b} \\
& -\left(K_{r p i} \delta_{r p i L}+C_{r p i} \dot{\delta}_{r p i L}\right) \sin \beta_{b}+K_{p a} z_{p i L} \\
& +C_{p a} \dot{z}_{p i L}+K_{p L R}\left(z_{p i L}-z_{p i R}\right) \\
& +C_{p L R}\left(\dot{z}_{p i L}-\dot{z}_{p i R}\right)=0 \\
& \frac{I_{p}}{r_{p i}} \ddot{u}_{z p i L}+\left(K_{s p i} \delta_{s p i L}+C_{s p i} \dot{\delta}_{s p i L}\right) r_{p i} \cos \beta_{b} \\
& -\left(K_{r p i} \delta_{r p i L}+C_{r p i} \dot{\delta}_{r p i L}\right) r_{p i} \cos \beta_{b} \\
& +K_{2 t}\left(u_{p i L}-u_{p i R}\right)+C_{2 t}\left(\theta_{p i L}-\theta_{p i R}\right)=0, \\
& m_{p}\left(\ddot{x}_{p i R}-2 \omega_{c} \dot{y}_{p i R}-\omega_{c}^{2} x_{p i R}\right) \\
& -\left(K_{s p i} \delta_{s p i R}+C_{s p i} \dot{\delta}_{s p i R}\right) \sin \alpha_{s n} \cos \beta_{b} \\
& +\left(K_{r p i} \delta_{r p i R}+C_{r p i} \dot{\delta}_{r p i R}\right) \sin \alpha_{r n} \cos \beta_{b}+K_{p} x_{p i R} \\
& +C_{p} \dot{x}_{p i R}=0 \\
& m_{p}\left(\ddot{y}_{p i R}+2 \omega_{c} \dot{x}_{p i R}-\omega_{c}^{2} y_{p i R}\right) \\
& \text { - }\left(K_{s p i} \delta_{s p i R}+C_{s p i} \dot{\delta}_{s p i R}\right) \cos \alpha_{s n} \cos \beta_{b} \\
& -\left(K_{r p i} \delta_{r p i R}+C_{r p i} \dot{\delta}_{r p i R}\right) \cos \alpha_{r n} \cos \beta_{b} \\
& +K_{p}\left(y_{p i R}-r_{c} u_{c}\right)+C_{p}\left(\dot{y}_{p i R}-r_{c} \dot{u}_{c}\right)=0 \\
& m_{p} \ddot{z}_{p i R}-\left(K_{s p i} \delta_{s p i R}+C_{s p i} \dot{\delta}_{s p i R}\right) \cos \beta_{b} \\
& +\left(K_{r p i} \delta_{r p i R}+C_{r p i} \dot{\delta}_{r p i R}\right) \cos \beta_{b}+K_{p a} z_{p i R} \\
& +C_{p a} \dot{z}_{p i R}+K_{p L R}\left(z_{p i R}-z_{p i L}\right) \\
& +C_{p L R}\left(\dot{z}_{p i R}-\dot{z}_{p i L}\right)=0 \\
& \frac{I_{p}}{r_{p i}} \ddot{u}_{z p i R}+\left(K_{s p i} \delta_{s p i R}+C_{s p i} \dot{\delta}_{s p i R}\right) r_{p i} \cos \beta_{b} \\
& -\left(K_{r p i} \delta_{r p i R}+C_{r p i} \dot{\delta}_{r p i R}\right) r_{p i} \cos \beta_{b} \\
& +K_{2 t}\left(u_{p i R}-u_{p i L}\right)+C_{2 t}\left(\theta_{p i R}-\theta_{p i L}\right)=0 \text {, }
\end{aligned}
$$

where $m_{p}$ is the equivalent mass of planetary gear, $K_{p}$ is the radial support stiffness of planetary gear, $C_{p}$ is the radial support damping of planetary gear, $K_{p a}$ is the axial support 
stiffness, $C_{p a}$ is the axial support damping, $K_{p 12}$ is the axial stiffness between two helical gears, $C_{p 12}$ is the axial damping between two helical gears, $K_{2 t}$ is the left-right coupling torsional stiffness, and $C_{2 t}$ is the left-right coupling torsional damping.

The axial support stiffness and support damping of planetary gear are as follows:

$$
\begin{aligned}
& K_{p a}=2 K_{p} \sin \beta \\
& C_{p a}=2 C_{p} \sin \beta .
\end{aligned}
$$

The differential equation of the dynamics of ring gear can be written as (13) and (14). Equation (13) is the dynamics equation of the left side of ring gear and (14) is the dynamics equation of the right side of ring gear.

$$
\begin{aligned}
& m_{r}\left(\ddot{x}_{r L}-2 \omega_{c} \dot{y}_{r L}-\omega_{c}^{2} x_{r L}\right) \\
& -\sum_{i=1}^{N}\left(K_{r p i} \delta_{r p i L}+C_{r p i} \dot{\delta}_{r p i L}\right) \sin \left(\phi_{r}-\alpha_{r p i}\right) \cos \beta_{b} \\
& +K_{r} x_{r L}+C_{r} \dot{x}_{r L}=0 \\
& m_{r}\left(\ddot{y}_{r L}+2 \omega_{c} \dot{x}_{r L}-\omega_{c}^{2} y_{r L}\right) \\
& +\sum_{i=1}^{N}\left(K_{r p i} \delta_{r p i L}+C_{r p i} \dot{\delta}_{r p i L}\right) \cos \left(\phi_{r}-\alpha_{r p i}\right) \cos \beta_{b} \\
& +K_{r} y_{r L}+C_{r} \dot{y}_{r L}=0 \\
& m_{r} \ddot{z}_{r L}+\sum_{i=1}^{N}\left(K_{r p i} \delta_{r p i L}+C_{r p i} \dot{\delta}_{r p i L}\right) \sin \beta_{b}+K_{r a} z_{r L} \\
& +C_{r a} \dot{z}_{r L}+K_{r L R}\left(z_{r L}-z_{r R}\right)+C_{r L R}\left(\dot{z}_{r L}-\dot{z}_{r R}\right)=0 \\
& \frac{I_{r}}{r_{r}} \ddot{u}_{z r L}+\sum_{i=1}^{N}\left(K_{r p i} \delta_{r p i L}+C_{r p i} \dot{\delta}_{r p i R}\right) r_{r} \cos \beta_{b} \\
& +K_{3 t}\left(u_{r L}-u_{r R}\right)+C_{3 t}\left(\theta_{r L}-\theta_{r R}\right)=0, \\
& m_{r}\left(\ddot{x}_{r R}-2 \omega_{c} \dot{y}_{r R}-\omega_{c}^{2} x_{r R}\right) \\
& -\sum_{i=1}^{N}\left(K_{r p i} \delta_{r p i R}+C_{r p i} \dot{\delta}_{r p i R}\right) \sin \left(\phi_{r}-\alpha_{r p i}\right) \cos \beta_{b} \\
& +K_{r} x_{r R}+C_{r} \dot{x}_{r R}=0 \\
& m_{r}\left(\ddot{y}_{r R}+2 \omega_{c} \dot{x}_{r R}-\omega_{c}^{2} y_{r R}\right) \\
& +\sum_{i=1}^{N}\left(K_{r p i} \delta_{r p i R}+C_{r p i} \dot{\delta}_{r p i R}\right) \cos \left(\phi_{r}-\alpha_{r p i}\right) \cos \beta_{b} \\
& +K_{r} y_{r R}+C_{r} \dot{y}_{r R}=0 \\
& m_{r} \ddot{z}_{r R}-\sum_{i=1}^{N}\left(K_{r p i} \delta_{r p i R}+C_{r p i} \dot{\delta}_{r p i R}\right) \sin \beta_{b}+K_{r a} z_{r R} \\
& +C_{r a} \dot{z}_{r R}+K_{r L R}\left(z_{r R}-z_{r L}\right)+C_{r L R}\left(\dot{z}_{r R}-\dot{z}_{r L}\right)=0
\end{aligned}
$$

$$
\begin{aligned}
& \frac{I_{r}}{r_{r}} \ddot{u}_{z r R}+\sum_{i=1}^{N}\left(K_{r p i} \delta_{r p i R}+C_{r p i} \dot{\delta}_{r p i R}\right) r_{r} \cos \beta_{b} \\
& \quad+K_{3 t}\left(u_{r R}-u_{r L}\right)+C_{3 t}\left(\theta_{r R}-\theta_{r L}\right)=0
\end{aligned}
$$

where $m_{r}$ is the equivalent mass of planetary gear, $K_{r}$ is the radial support stiffness of planetary gear, $C_{r}$ is the radial support damping of planetary gear, $K_{r a}$ is the axial support stiffness, $C_{r a}$ is the axial support damping, $K_{r 12}$ is the axial stiffness between two helical gears, $C_{r 12}$ is the axial damping between two helical gears, $K_{3 t}$ is the left-right coupling torsional stiffness, and $C_{3 t}$ is the left-right coupling torsional damping.

The axial support stiffness and support damping of ring gear are as follows:

$$
\begin{aligned}
& K_{r a}=2 K_{r} \sin \beta \\
& C_{r a}=2 C_{r} \sin \beta .
\end{aligned}
$$

Figure 4 is the dynamics model of carrier, which combined with the Newton second law can deduct the differential equation of the carrier dynamics, as shown in

$$
\begin{aligned}
m_{c} & \left(\ddot{x}_{c}-2 \omega_{c} \dot{y}_{c}-\omega_{c} x_{c}\right) \\
& -\sum_{n=1}^{N}\left[K_{p}\left(x_{p i L}+x_{p i R}\right)+C_{p}\left(\dot{x}_{p i L}+\dot{x}_{p i R}\right)\right] \\
& +K_{c} x_{c}+C_{c} \dot{y}_{c}=0 \\
m_{c} & \left(\ddot{y}_{c}+2 \omega_{c} \dot{x}_{c}-\omega_{c} y_{c}\right) \\
& -\sum_{n=1}^{N}\left[K_{p}\left(y_{p i L}+y_{p i R}\right)+C_{p}\left(\dot{y}_{p i L}+\dot{y}_{p i R}\right)\right] \\
& +K_{c} y_{c}+C_{c} \dot{y}_{c}=0 \\
m_{c} \ddot{z}_{c} & +K_{c a} z_{c}+C_{c a} \dot{z}_{c} \\
& -\sum_{n=1}^{N}\left[K_{p a}\left(z_{p i L}+z_{p i R}\right)-C_{p a}\left(\dot{z}_{p i L}+\dot{z}_{p i R}\right)\right] \\
& +K_{c a} z_{c}+C_{c a} \dot{z}_{c}=0 \\
& +C_{c t} \theta_{z c}=T_{o u t}, \\
r_{c} & \ddot{u}_{z c}+\sum_{i=1}^{n} K_{p} r_{c}\left(2 u_{z c} r_{c}-y_{p i L}-y_{p i R}\right)+K_{c t} u_{z c} \\
&
\end{aligned}
$$

where $m_{c}$ is the equivalent mass of carrier, $K_{c}$ is the radial support stiffness of carrier, $C_{c}$ is the radial support damping of carrier, $K_{c a}$ is the axial support stiffness, $C_{c a}$ is the axial support damping, $K_{c t}$ is the torsional stiffness, and $C_{c t}$ is the torsional damping.

The axial support stiffness and support damping of carrier is as follows:

$$
\begin{aligned}
& K_{c a}=2 K_{c} \sin \beta \\
& C_{c a}=2 C_{c} \sin \beta
\end{aligned}
$$




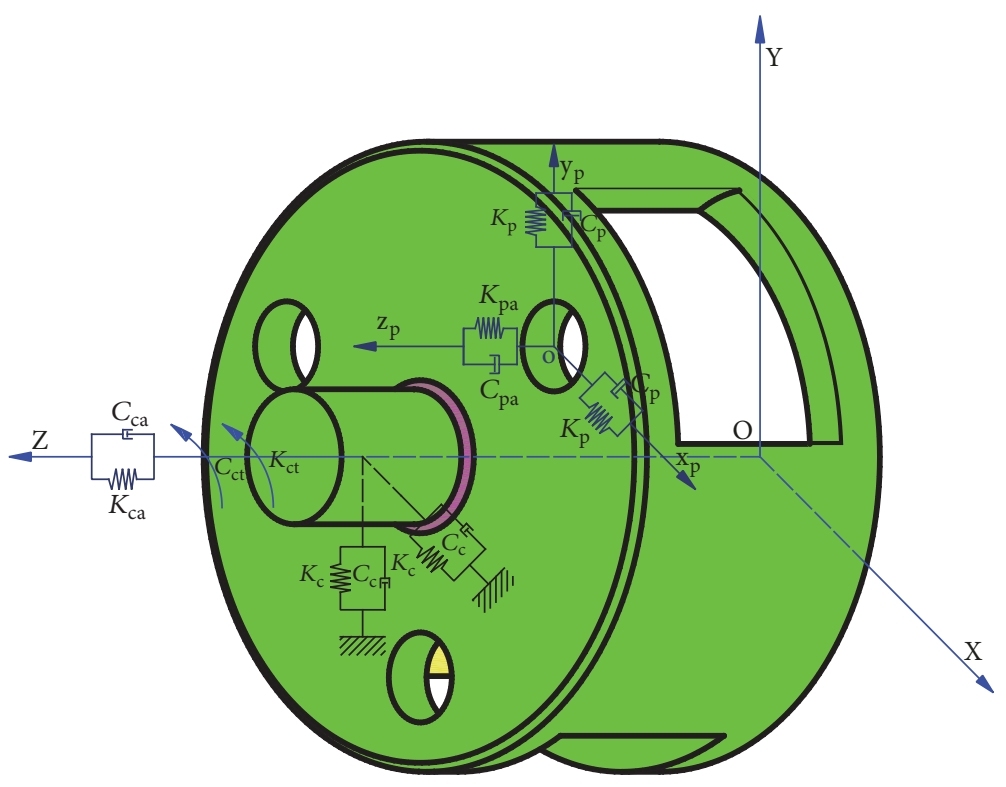

FIgURE 4: Dynamics model of carrier.

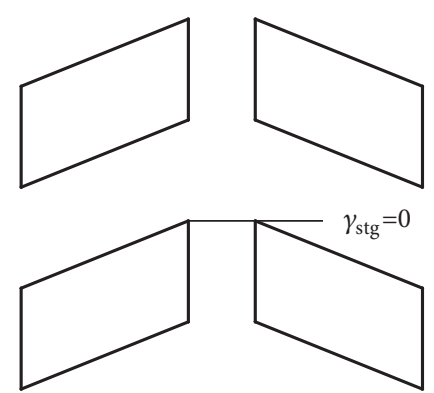

(a) Sketch of stagger angle is 0

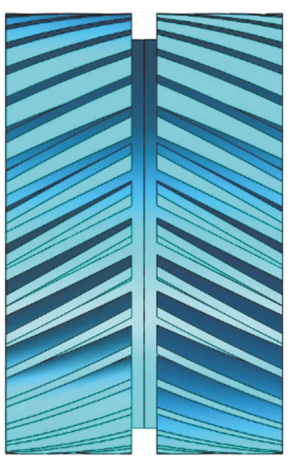

FIgURE 5: The model sketch of stagger angle.
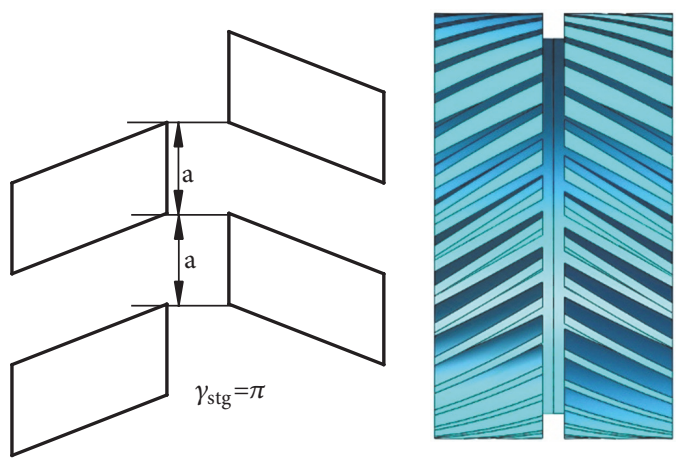

(b) Sketch of stagger angle is $\pi$ 


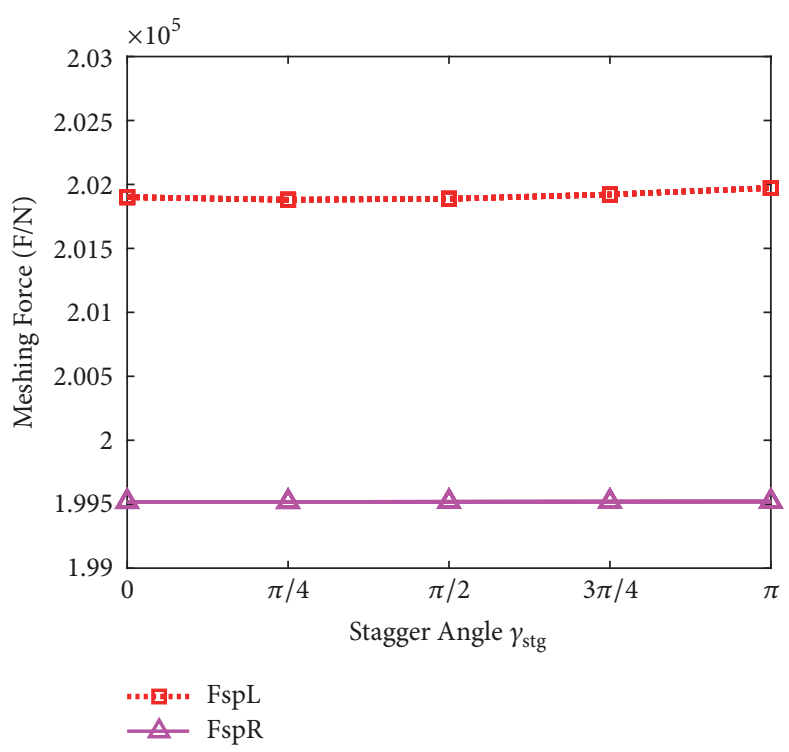

(a) Inner meshing

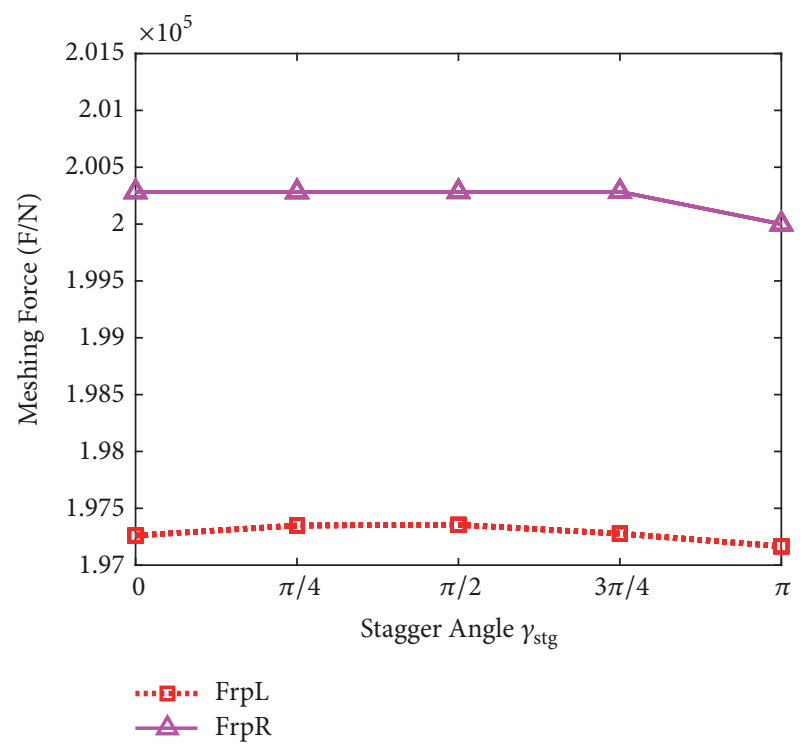

(b) Outer meshing

FIGURE 6: Curve of maximum meshing force-stagger angle.

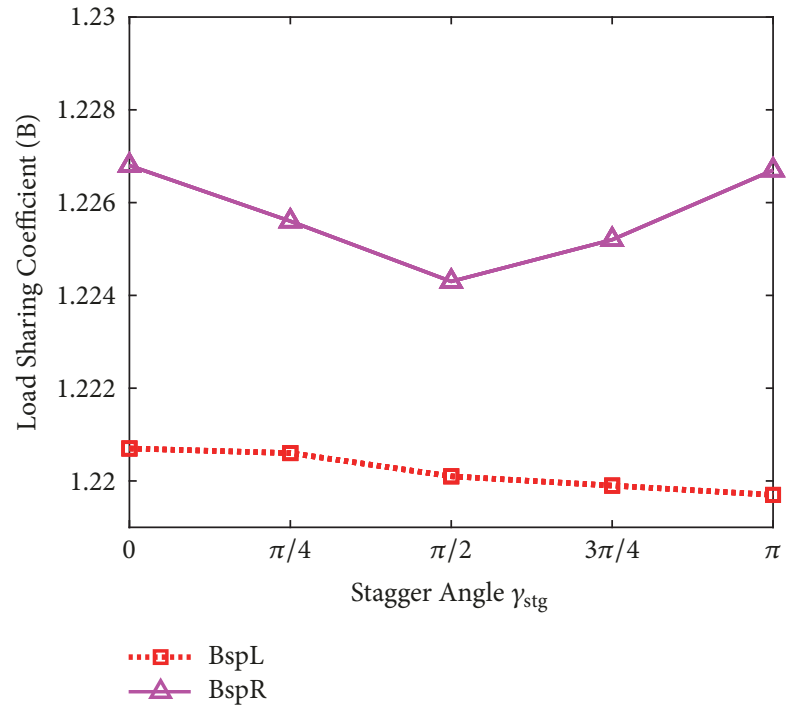

(a) Inner meshing

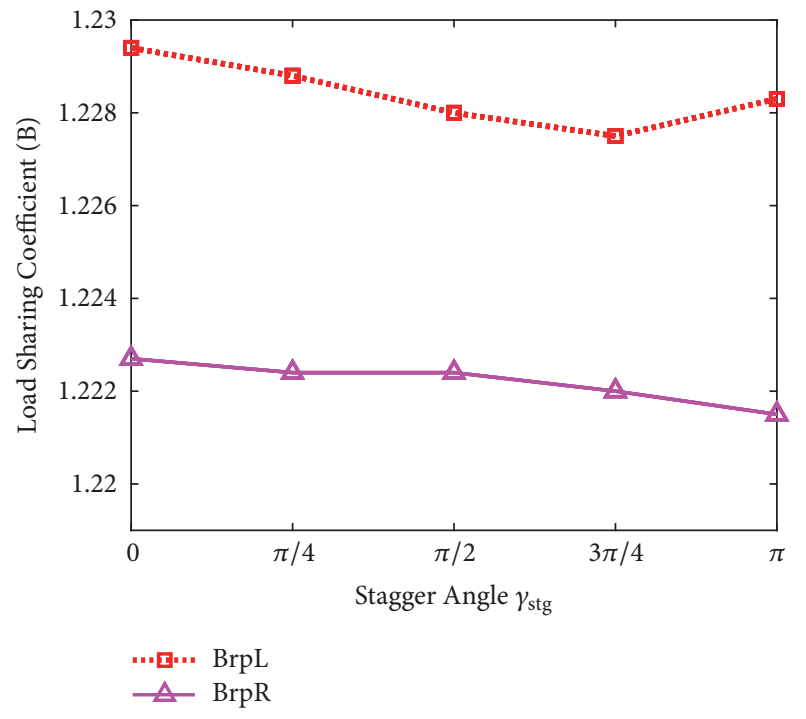

(b) Outer meshing

FIGURE 7: Curve of load sharing coefficient-stagger angle.

Error excitation of inner meshing is

$$
\begin{aligned}
& e_{s p i}^{(L)}(t)=\sum_{l=1}^{L} \widehat{e}_{s p l} \cos \left(l \omega_{m} t+\sigma_{s p l}-l z_{s} \varphi_{i}\right) \\
& e_{s p i}^{(R)}(t)=\sum_{l=1}^{L} \widehat{e}_{s p l} \cos \left(l \omega_{m} t+\sigma_{s p l}-l z_{s} \varphi_{i}+l \gamma_{s t g}\right) .
\end{aligned}
$$

And error excitation of outer meshing is

$$
\begin{aligned}
& e_{r p i}^{(L)}(t)=\sum_{l=1}^{L} \widehat{e}_{r p l} \cos \left(l \omega_{m} t+\sigma_{r p l}+l \gamma_{r s}+l z_{r} \varphi_{i}\right) \\
& e_{r p i}^{(R)}(t) \\
& =\sum_{l=1}^{L} \widehat{e}_{r p l} \cos \left(l \omega_{m} t+\sigma_{r p l}+l \gamma_{r s}+l z_{r} \varphi_{i}+l \gamma_{s t g}\right),
\end{aligned}
$$




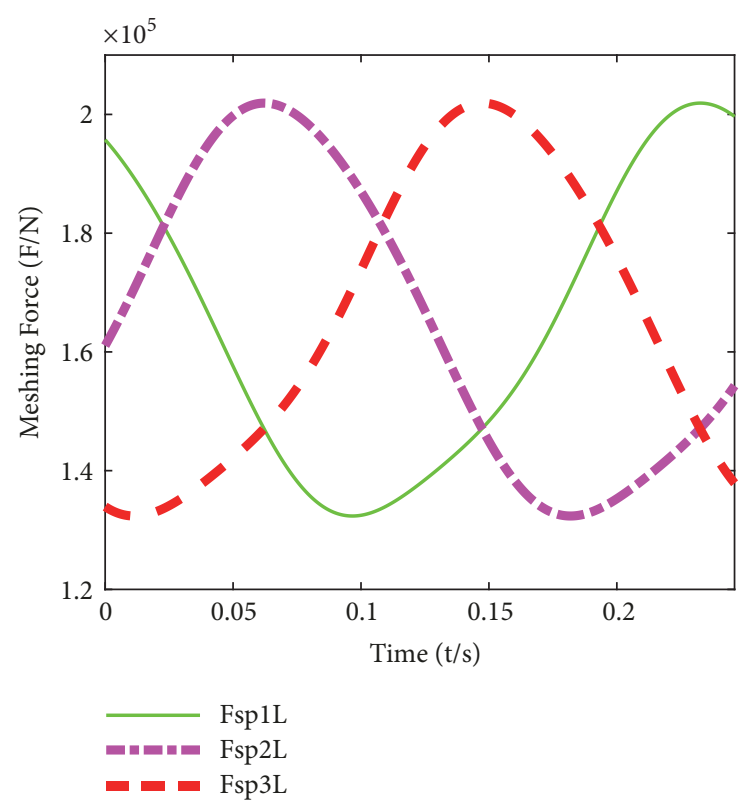

(a) Left-side meshing force of inner mesh

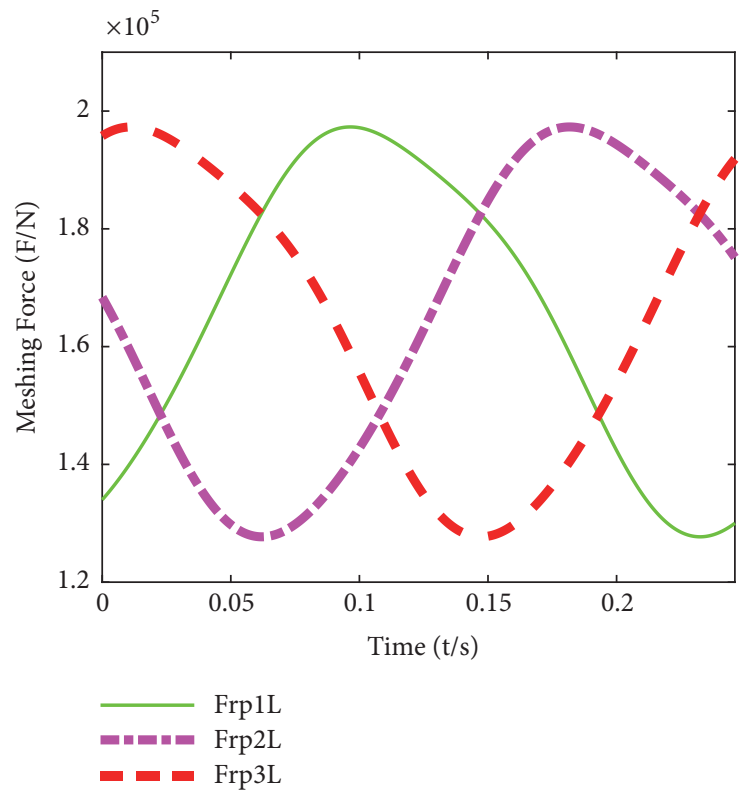

(c) Left-side meshing force of outer mesh

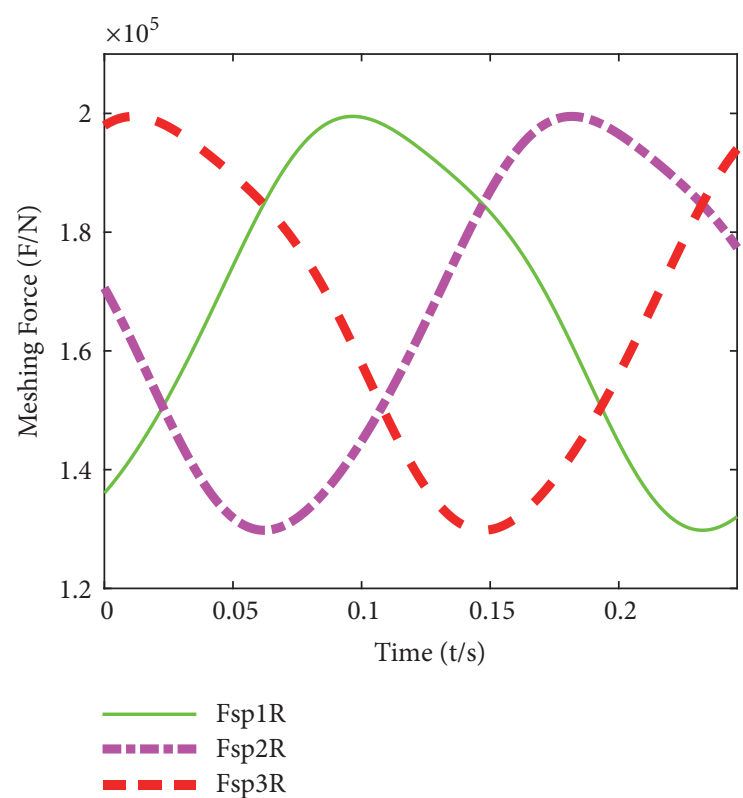

(b) Right-side meshing force of inner mesh

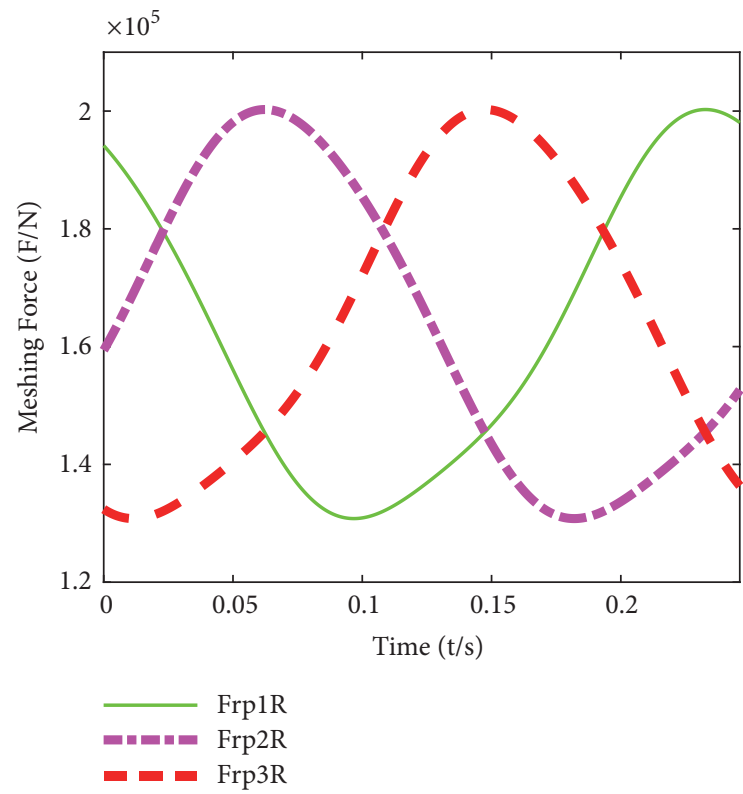

(d) Right-side meshing force of outer mesh

FIgURE 8: Meshing force curves of initial parameters.

where $\widehat{e}_{s p l}$ is the 1st order amplitude of the transfer error between the sun gear and the planetary gear, $\sigma_{s p l}$ is the initial phase of the transfer error between the sun gear and the planetary gear, $\widehat{e}_{r p l}$ is the 1st order amplitude of the transfer error between the sun gear and the planetary gear, $\sigma_{r p l}$ is the initial phase of the transfer error between the sun gear and the planetary gear, $\gamma_{r s}$ is the phase difference between the meshing of ring gear and planetary gear and the meshing of sun gear and planetary gear, and $\gamma_{s t g}$ is the stagger angle of herringbone gear.

\section{Impact of Stagger Angle on Dynamic Characteristic of System}

There is a wind power gearbox whose main parameters are shown in Table 1. And the initial amplitude and phase of gear transmission error can be calculated in Kisssoft. The numerical value of first 3-order harmonics amplitude, shown in Table 2, is needed because the numerical value of higher order harmonics amplitude is very small. 


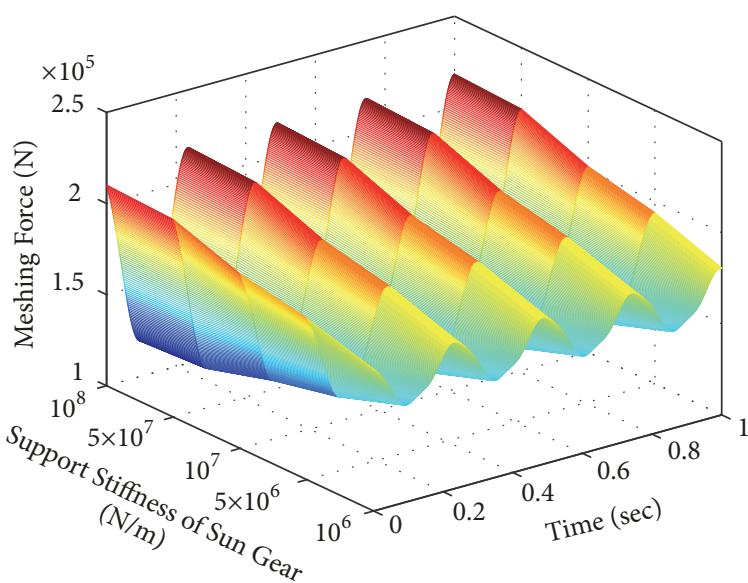

(a) 3D curve of left-side meshing force

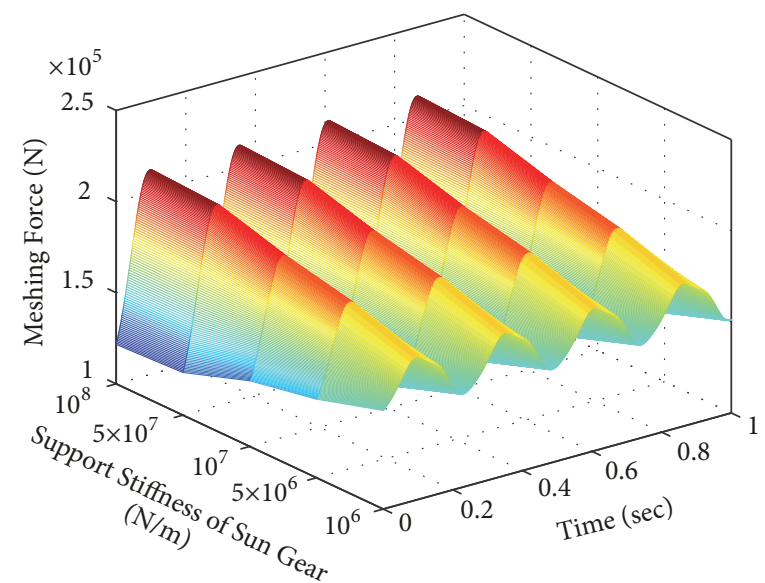

(b) 3D curve of right-side meshing force

FiguRE 9: Three-dimensional curves of inner meshing of planetary gear 1.

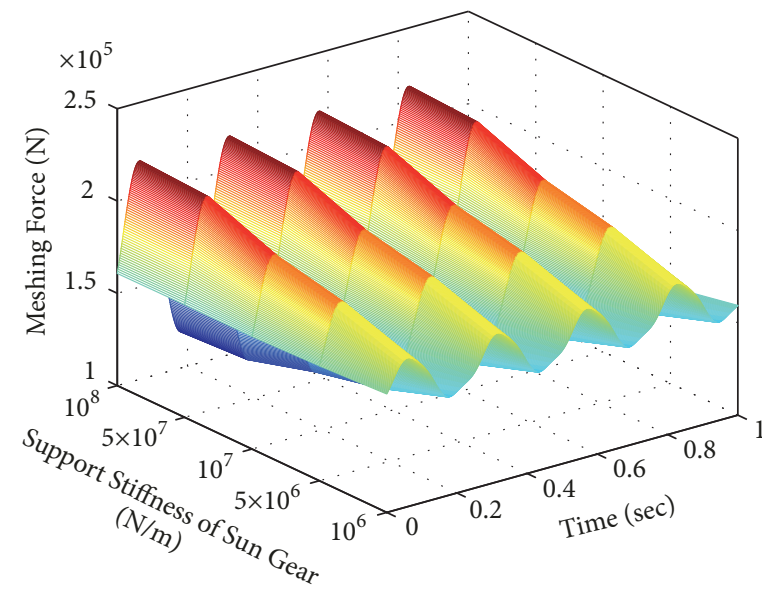

(a) Left-side meshing force $3 \mathrm{D}$ curve

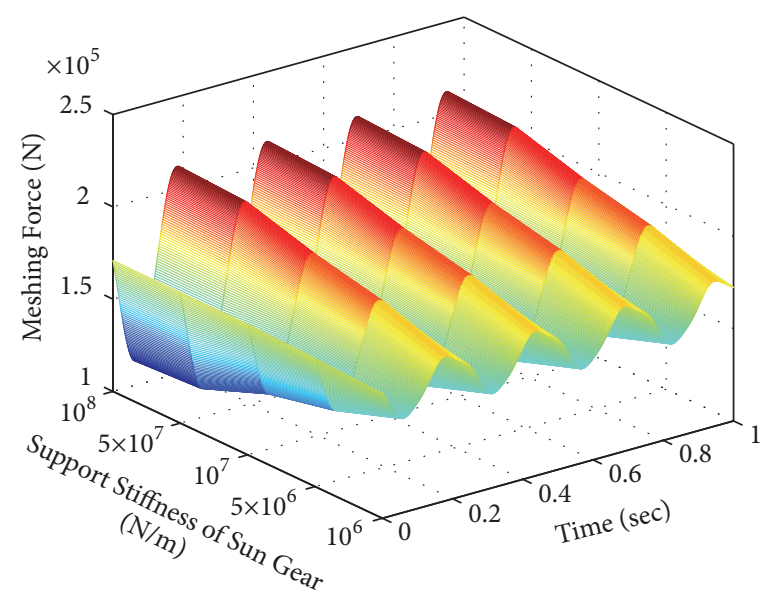

(b) Right-side meshing force 3D curve

FIGURE 10: Three-dimensional curves of inner meshing of planetary gear 2.

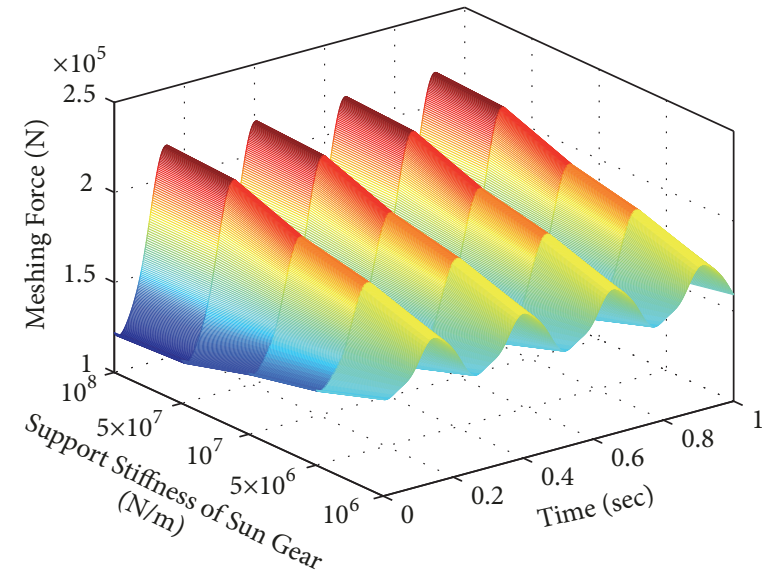

(a) 3D curve of left-side meshing force

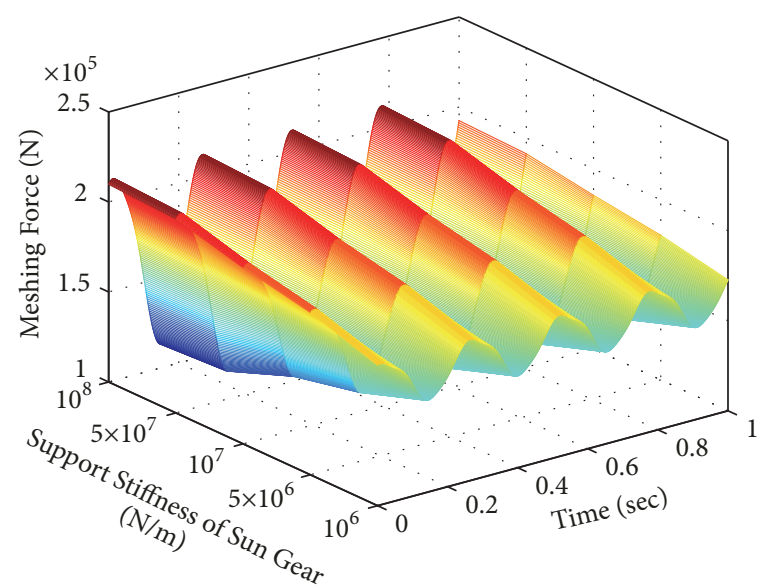

(b) 3D curve of right-side meshing force

FIgURE 11: Three-dimensional curves of inner meshing of planetary gear 3. 


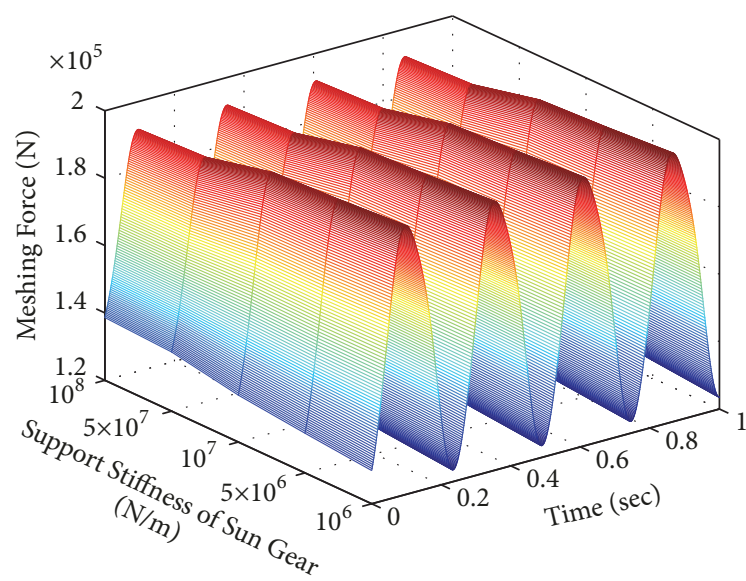

(a) 3D curve of left-side meshing force

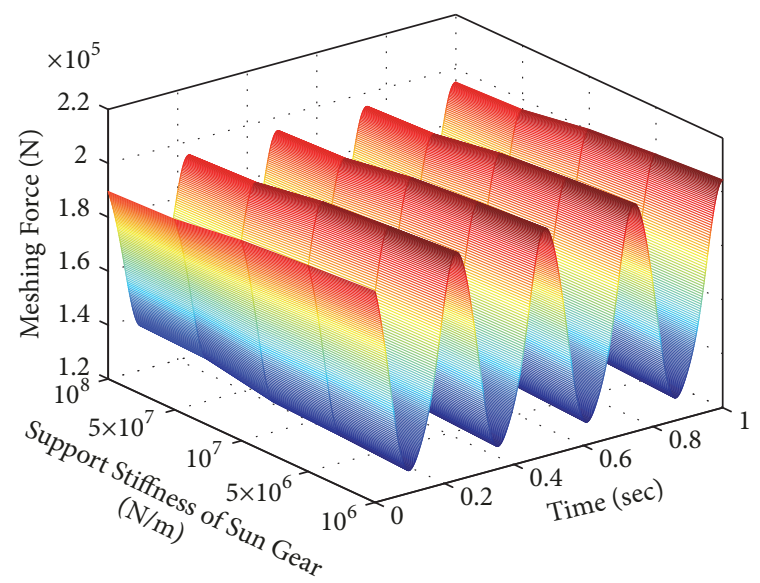

(b) 3D curve of right-side meshing force

FIGURE 12: Three-dimensional curves of outer meshing of planetary gear 1.

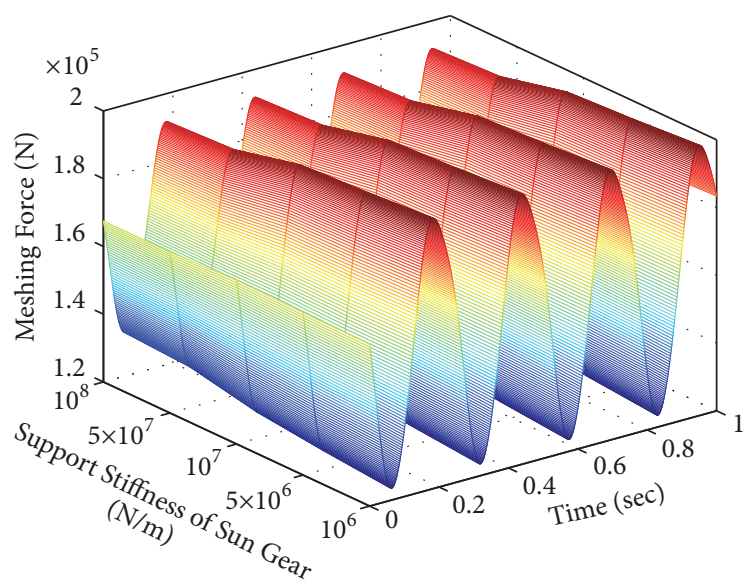

(a) 3D curve of left-side meshing force

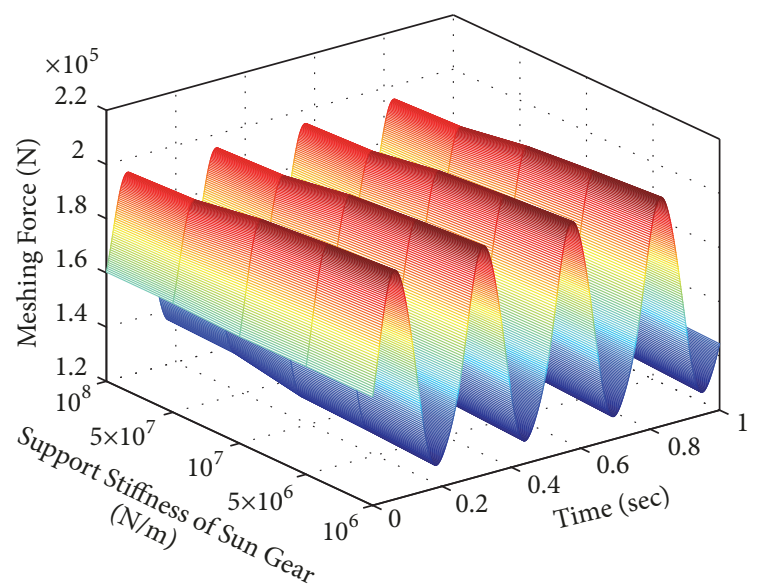

(b) 3D curve of right-side meshing force

FIGURE 13: Three-dimensional curves of outer meshing of planetary gear 2.

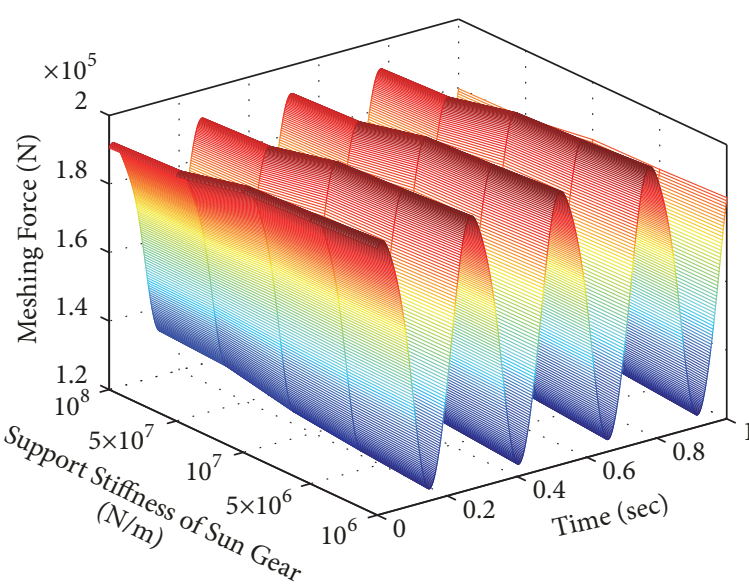

(a) 3D curve of left-side meshing force

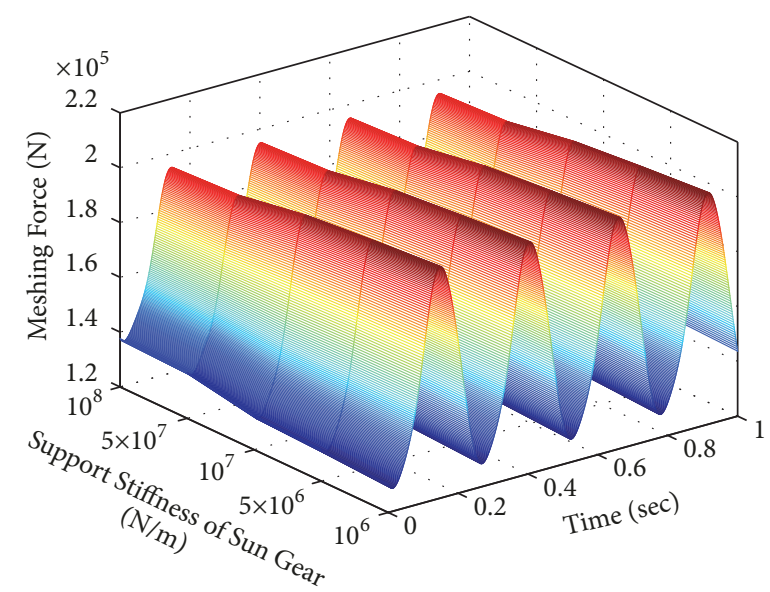

(b) 3D curve of right-side meshing force

FIGURE 14: Three-dimensional curves of outer meshing of planetary gear 3. 


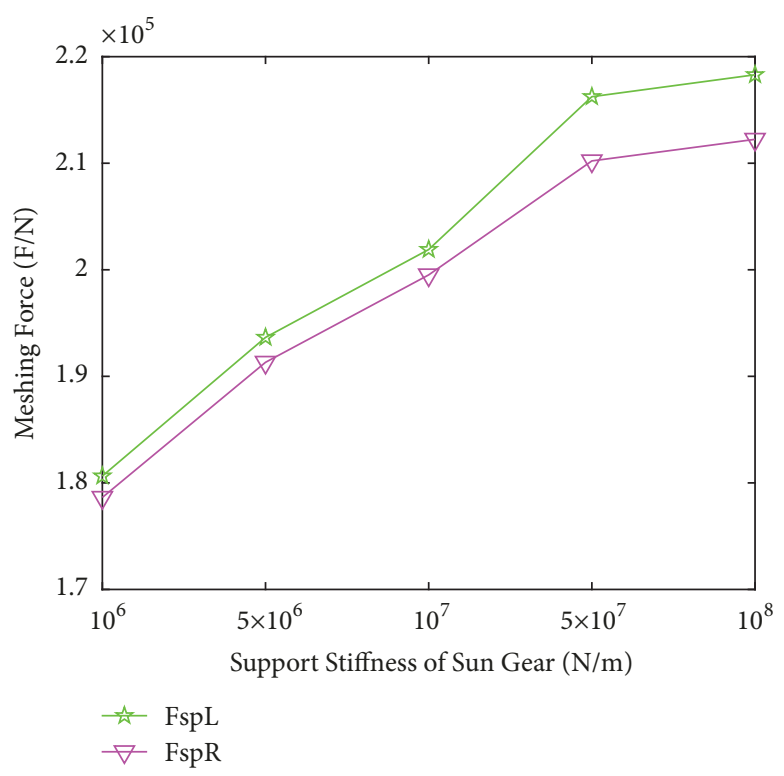

(a) Inner meshing maximum meshing force curve

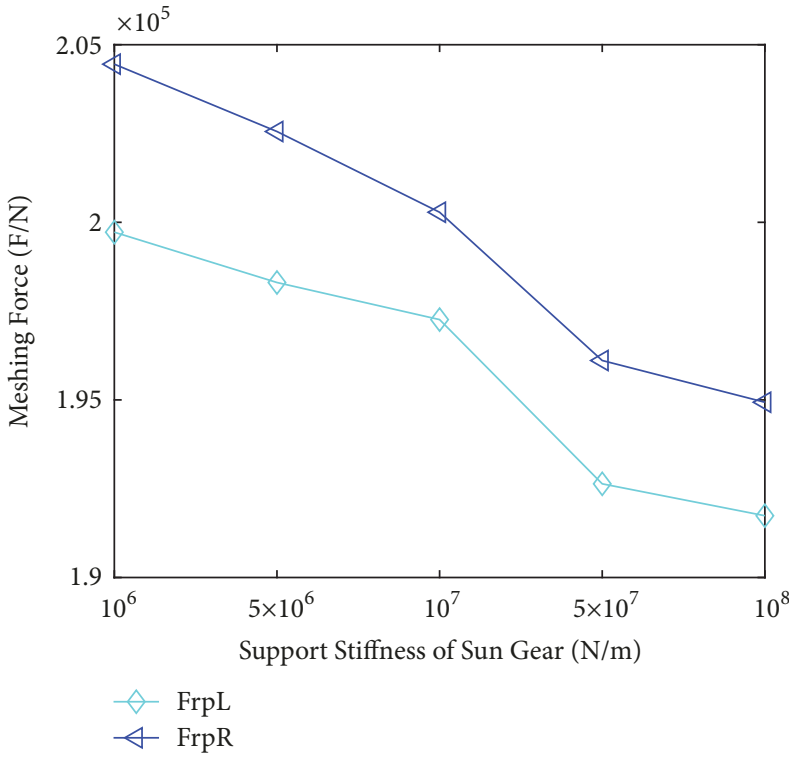

(b) Outer meshing maximum meshing force curve

FIGURE 15: Curves of maximum meshing force-support stiffness of sun gear.

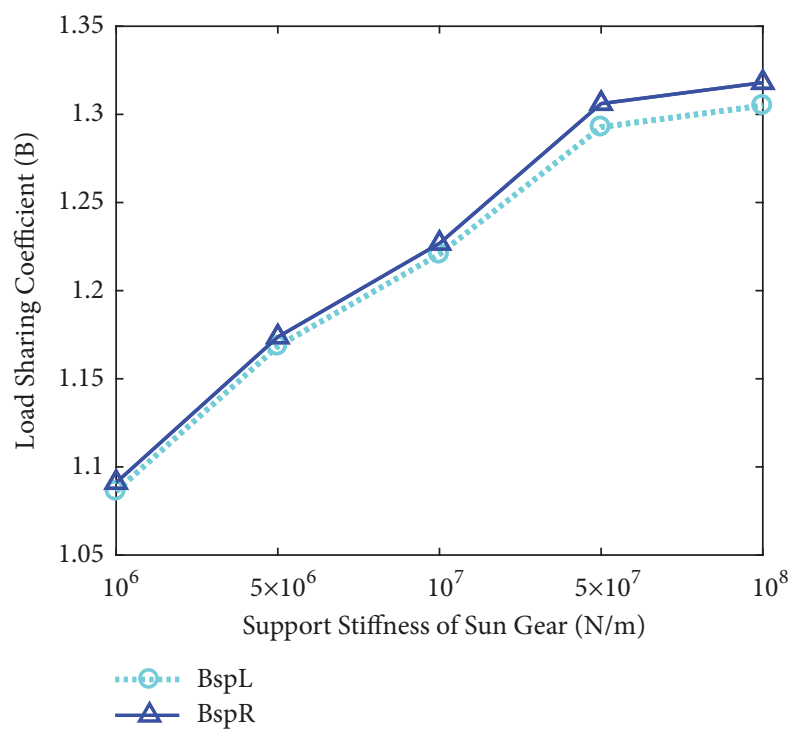

(a) Inner meshing load sharing coefficient curve

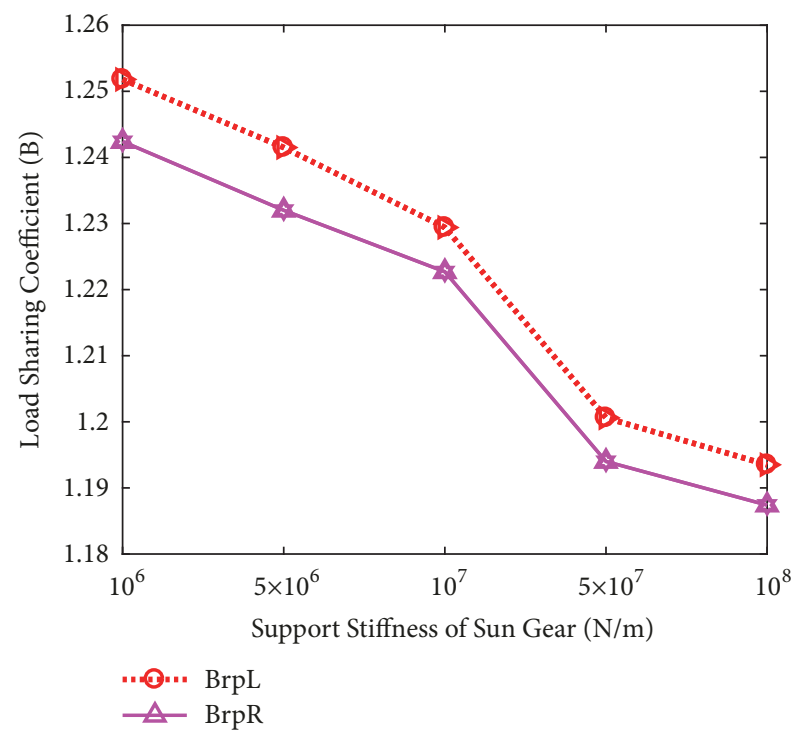

(b) Outer meshing load sharing coefficient curve

FIGURE 16: Curves of load sharing coefficient-support stiffness of sun gear.

Because of the special tooth shape of herringbone gear, there will be formed unavoidable stagger angle in the processing of gear manufacturing. As Figure 5 shown, left side is the plan sketch of stagger angle while the right side is the 3D model of it.

While stagger angle, respectively, is $0, \pi / 4, \pi / 2,3 \pi / 4$, and $\pi$, the corresponding maximum meshing force can be calculated. Figure 6 shows the maximum meshing force curve.
According to the equation of load sharing coefficient, the load sharing coefficient of a single gear pair can be defined as

$$
\begin{aligned}
& b_{s p n}=\frac{N \times F_{s p n}}{\sum_{i=1}^{N} F_{s p n}} \\
& b_{r p n}=\frac{N \times F_{r p n}}{\sum_{i=1}^{N} F_{r p n}},
\end{aligned}
$$




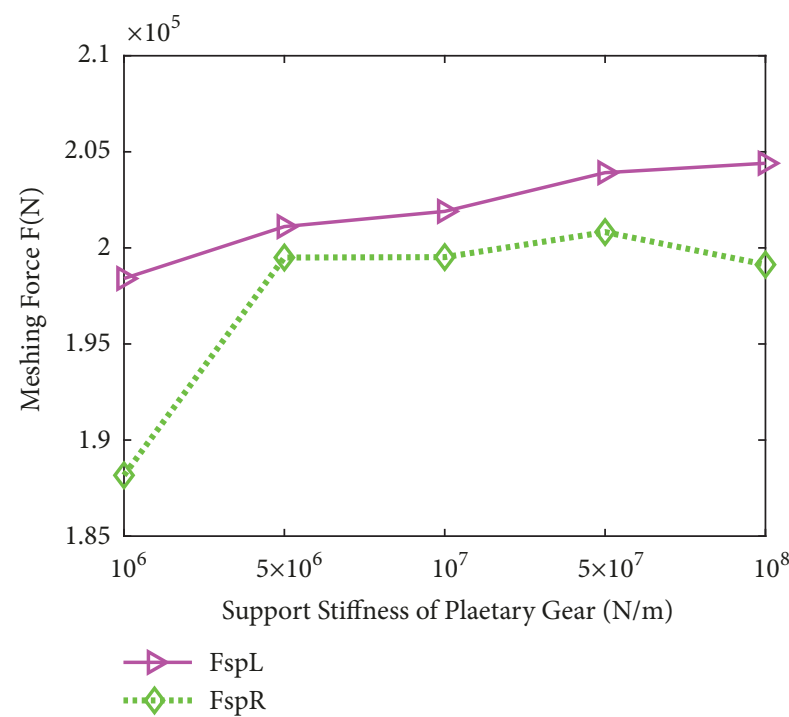

(a) Inner meshing maximum meshing force curve

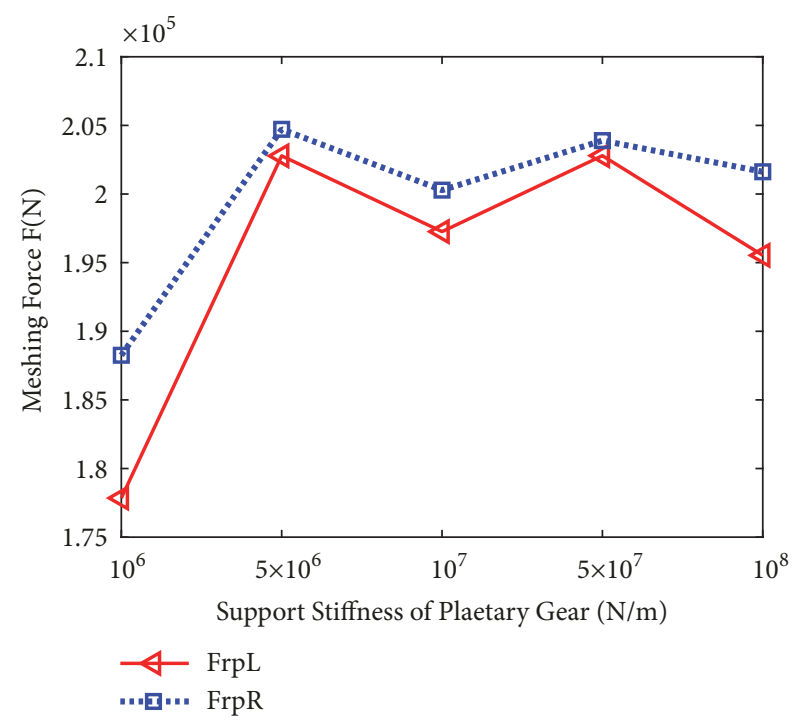

(b) Outer meshing maximum meshing force curve

FIGURE 17: Curves of maximum meshing force-support stiffness of planetary gear.

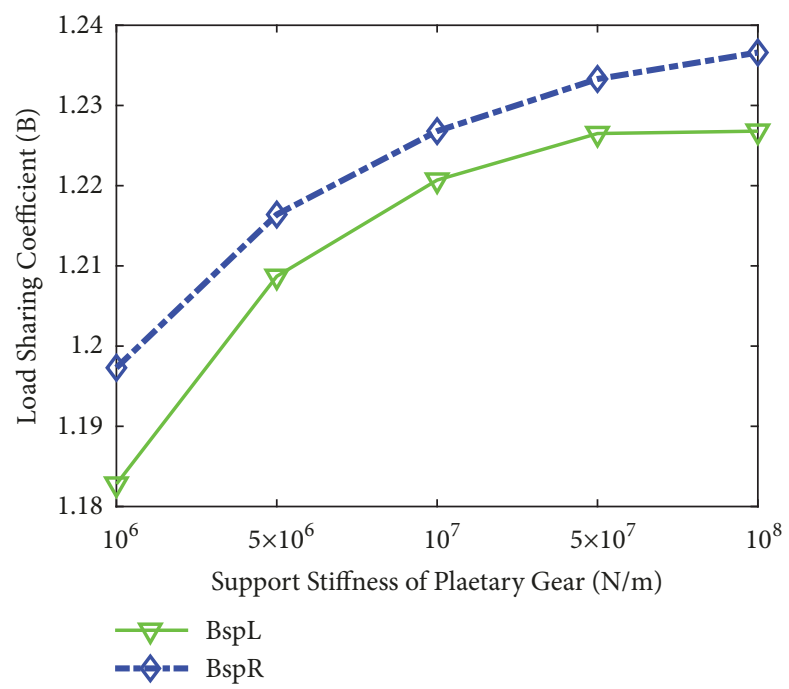

(a) Inner meshing load sharing coefficient curve

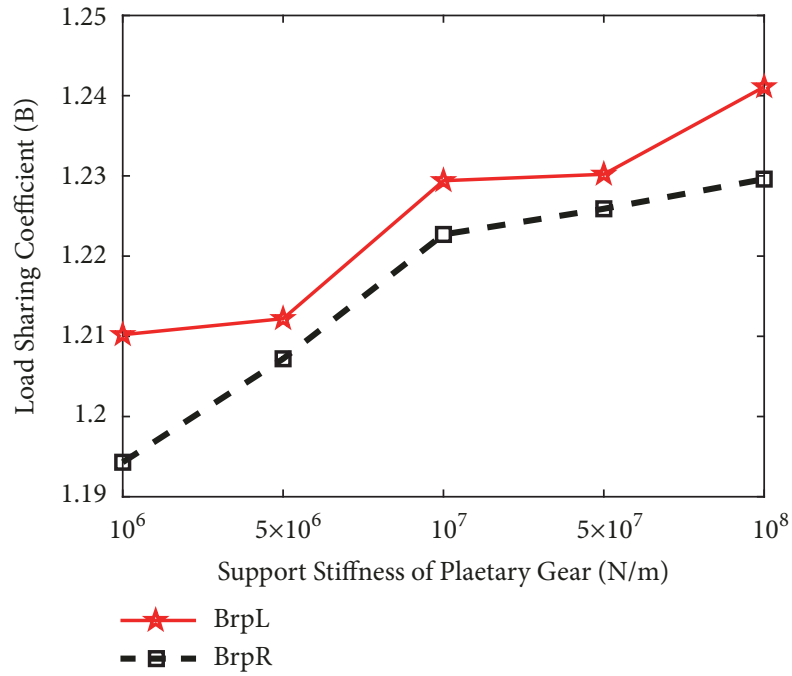

(b) Outer meshing load sharing coefficient curve

FIGURE 18: Curves of load sharing coefficient-support stiffness of planetary gear.

where $n$ is the period number of meshing frequency, $N$ is the number of planetary gear, and $F$ is the corresponding meshing force of the meshing cycle.

Likewise, the load sharing coefficient of herringbone gear inner and outer meshing is

$$
\begin{aligned}
& B_{s p}=\max \left(b_{s p n}\right) \\
& B_{r p}=\max \left(b_{r p n}\right) .
\end{aligned}
$$

The load sharing coefficient of inner and outer meshing can be calculated by (23), (24), and Figure 6, as shown in Figure 7.
In summary, the change of stagger angle has obvious influence on the dynamic characteristics of the left side of the herringbone gear but has little effect on the right side. In the processing of stagger angle increase from 0 to $\pi$, both the left and right side maximum meshing force have an unobvious change. But the load sharing coefficient of left side decreases at first and increases at last, while the right side decreases all the time. The load sharing coefficient of inner meshing meets the minimum when stagger angle is $\pi / 2$, and the outer meshing meets the minimum when stagger angle is $3 \pi / 4$. Overall factors which are comprehensively considered, the best stagger angle of this system is $3 \pi / 4$. 


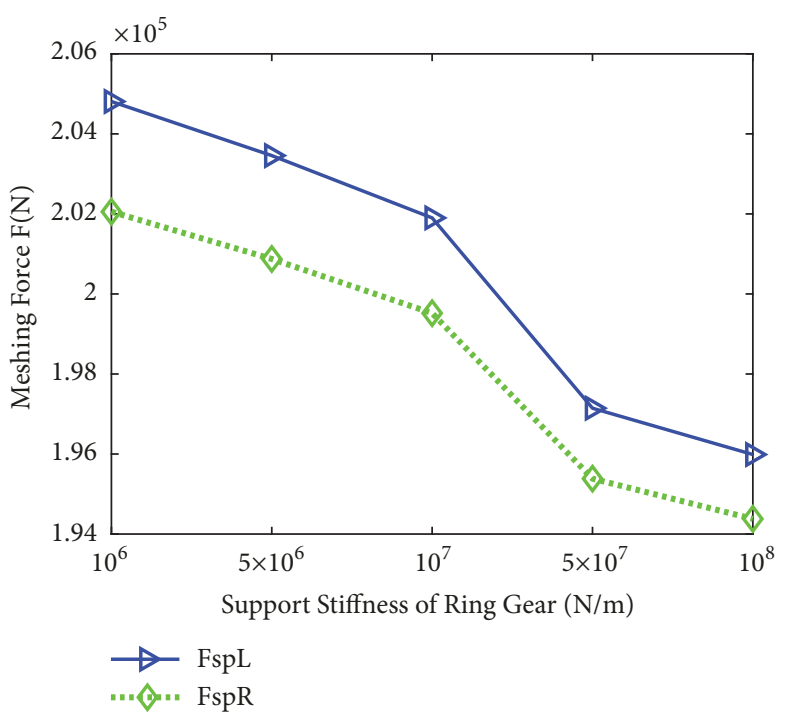

(a) Inner meshing maximum meshing force curve

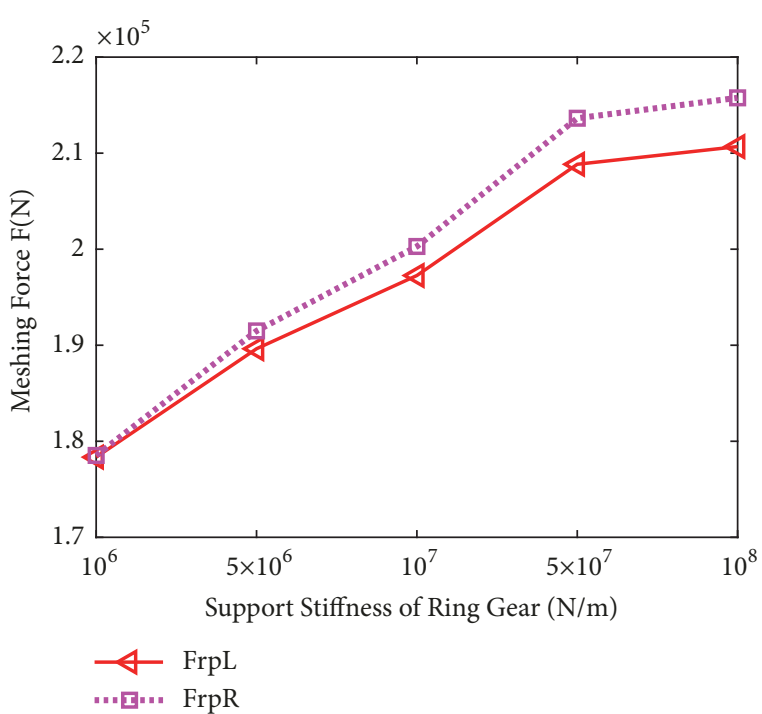

(b) Outer meshing maximum meshing force curve

FIGURE 19: Curves of maximum meshing force-support stiffness of ring gear.

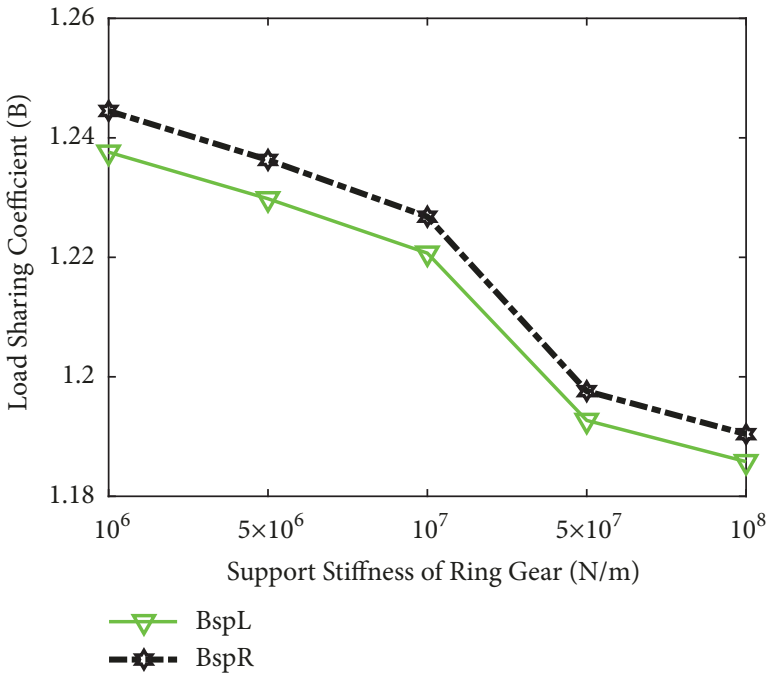

(a) Inner meshing load sharing coefficient curve

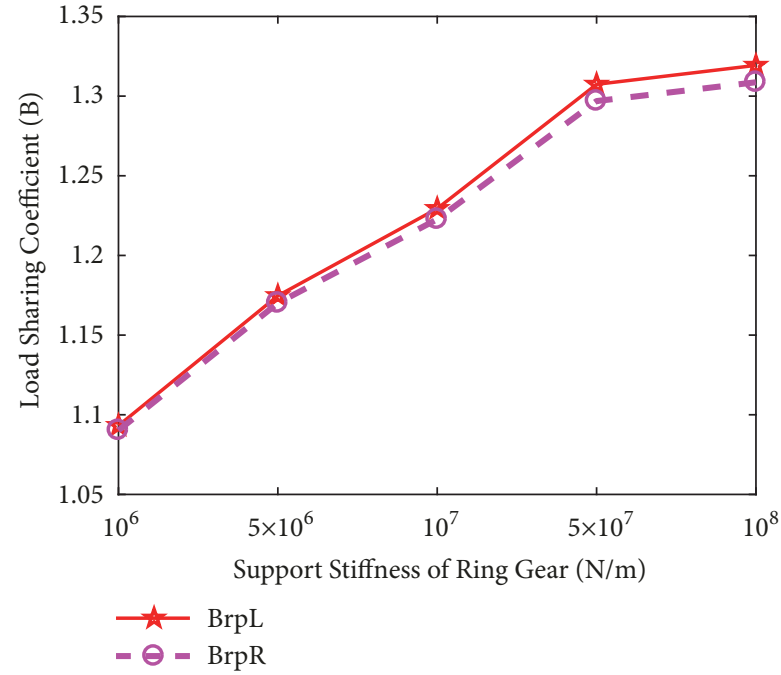

(b) Outer meshing load sharing coefficient curve

FIGURE 20: Curves of load sharing coefficient-support stiffness of ring gear.

\section{Impact of Support Stiffness on Dynamic Characteristic of System}

4.1. Theoretical Dynamics Analysis on Initial Parameter. To analyze the impact of support stiffness on dynamic characteristics of the herringbone gear planetary transmission system from the view of practical application, the parameters researched in this paper were gotten from the wind power gearbox described. In the transmission system, all types of the initial support stiffness of sun gear, planetary gear, and ring gear are $1 \times 10^{7} \mathrm{~N} / \mathrm{m}$, and the stagger angle is 0 . All of these parameters are substituted in the dynamics program; the meshing force is calculated and shown in Figure 8.
4.2. Theoretical Dynamics Analysis on Changing Support Stiffness. Keep the stiffness of planetary gear and ring gear be a constant value which is $10^{7} \mathrm{~N} / \mathrm{m}$, and change the stiffness of sun gear from $10^{6} \mathrm{~N} / \mathrm{m}$ to $10^{8} \mathrm{~N} / \mathrm{m}$. The stiffness, respectively, is $10^{6}, 5 \times 10^{6}, 10^{7}, 5 \times 10^{7}$, and $10^{8} \mathrm{~N} / \mathrm{m}$, and the corresponding meshing force can be gotten and be coupled as $3 \mathrm{D}$ sketch as shown in Figures 9-14.

The maximum meshing force of corresponding support stiffness of sun gear can be coupled as shown in Figure 15. Therefore, the load sharing coefficient curve is shown in Figure 16.

Likewise, keep the stiffness of sun gear and ring gear be a constant value which are $10^{7} \mathrm{~N} / \mathrm{m}$, and change the stiffness 


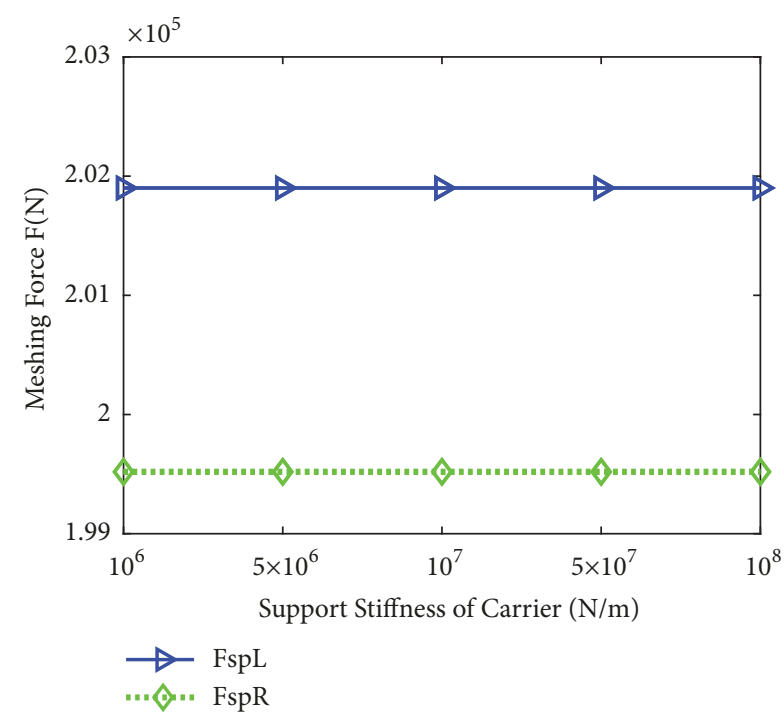

(a) Inner meshing maximum meshing force curve

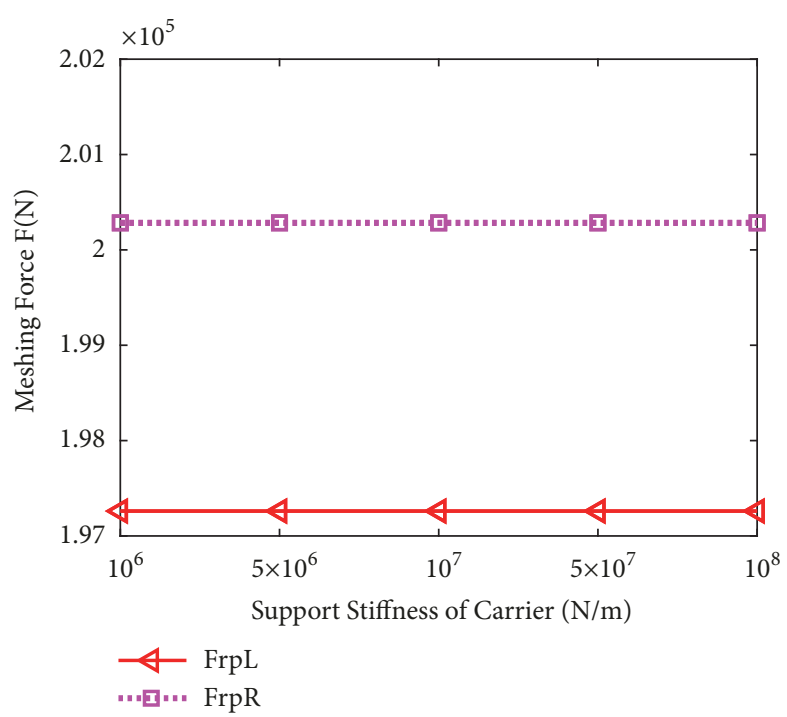

(b) Outer meshing maximum meshing force curve

FIGURE 21: Curves of maximum meshing force-support stiffness of carrier.

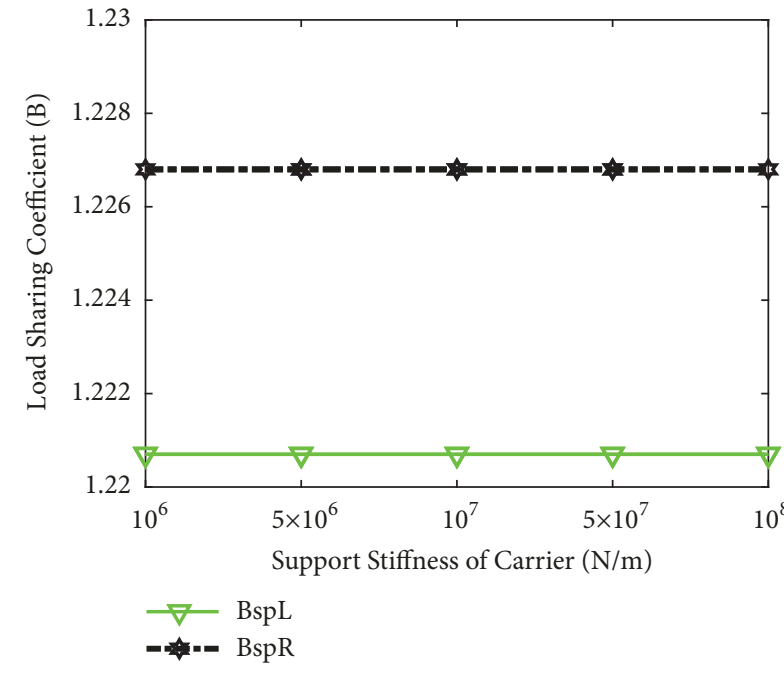

(a) Inner meshing load sharing coefficient curve

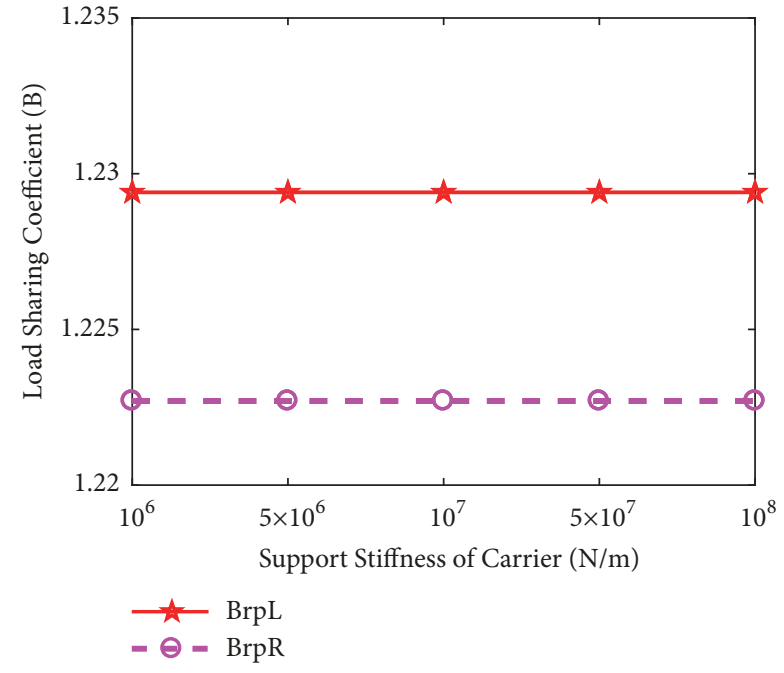

(b) Outer meshing load sharing coefficient curve

FIGURE 22: Curves of load sharing coefficient-support stiffness of carrier.

of planetary gear from $10^{6} \mathrm{~N} / \mathrm{m}$ to $10^{8} \mathrm{~N} / \mathrm{m}$. The stiffness is $10^{6}, 5 \times 10^{6}, 10^{7}, 5 \times 10^{7}$, and $10^{8} \mathrm{~N} / \mathrm{m}$, respectively. And the corresponding maximum meshing force and load sharing coefficient can be gotten as shown in Figures 17 and 18. Keep the stiffness of sun gear and planetary gear be a constant value which are $10^{7} \mathrm{~N} / \mathrm{m}$, and change the stiffness of ring gear from $10^{6} \mathrm{~N} / \mathrm{m}$ to $10^{8} \mathrm{~N} / \mathrm{m}$. The corresponding maximum meshing force and load sharing coefficient can be shown in Figures 19 and 20 .

And change the support stiffness of carrier while the support stiffness of gears is a constant value, as shown in
Figures 21 and 22. According to the research results, commendatory support stiffness of the studied system is as follows: support stiffness of sun gear $10^{7} \mathrm{~N} / \mathrm{m}$, support stiffness of planetary gear $10^{7} \mathrm{~N} / \mathrm{m}$, support stiffness of ring gear $10^{7} \mathrm{~N} / \mathrm{m}$, and support stiffness of carrier $10^{7} \mathrm{~N} / \mathrm{m}$. Curves of meshing fore and load sharing coefficient with commendatory support stiffness are shown in Figures 23 and 24, where load sharing coefficient is written as LSC.

In summary, firstly, with the increase of the support stiffness of sun gear, both of the maximum meshing force and load sharing coefficient of inner meshing increase 


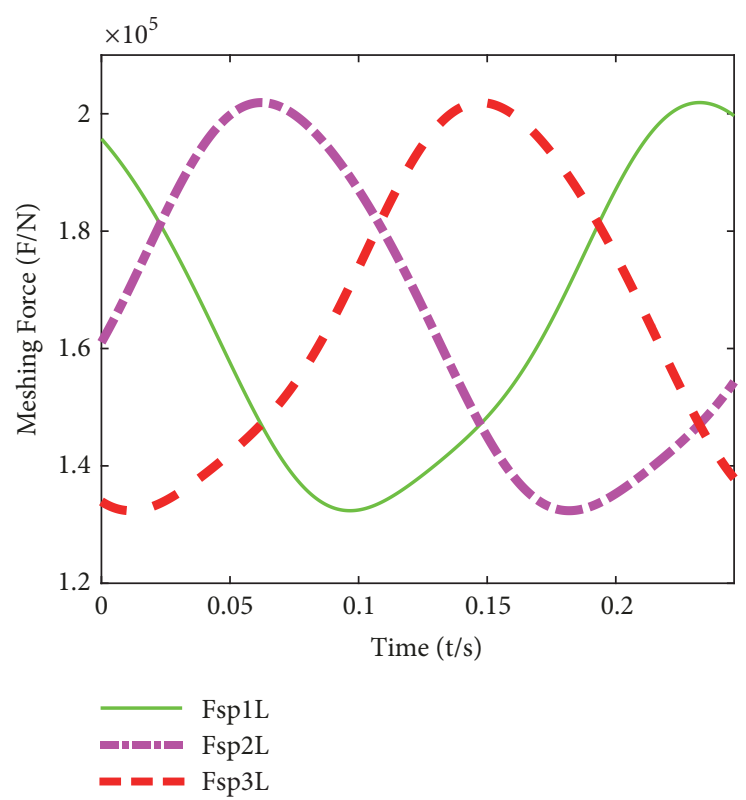

(a) The left-side meshing force of inner mesh

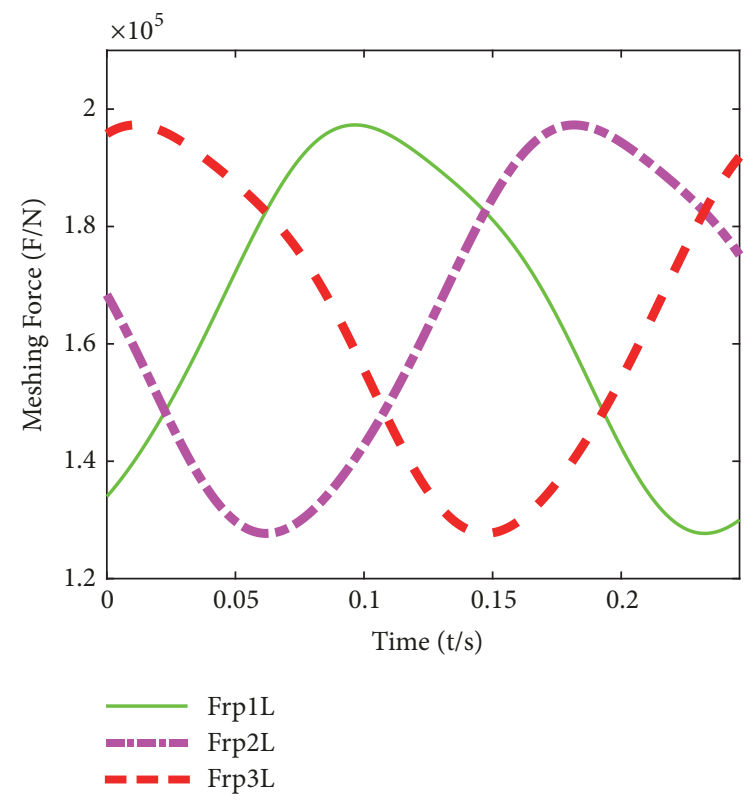

(c) The left-side meshing force of outer mesh

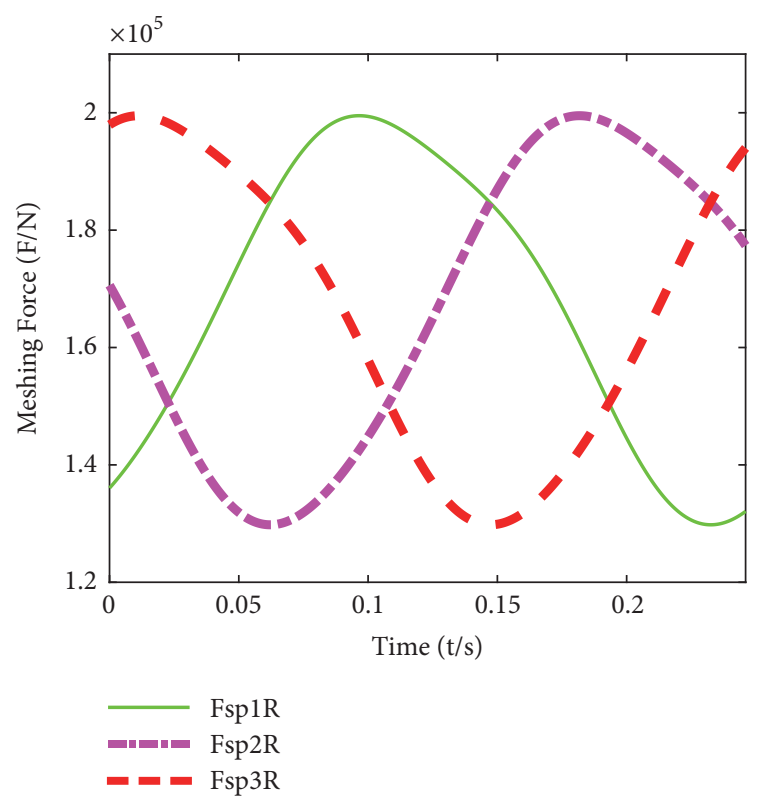

(b) The right-side meshing force of inner mesh

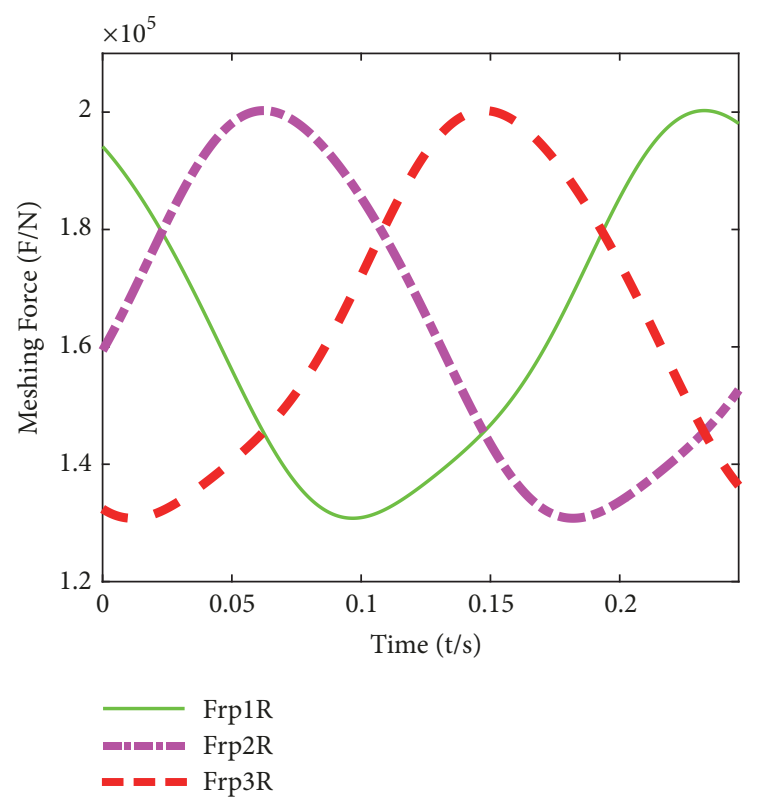

(d) The right-side meshing force of outer mesh

FIGURE 23: Meshing force curves of commendatory parameter.

while these of outer meshing decrease. Secondly, with the increase of support stiffness of planetary gear, both of the maximum meshing force and load sharing coefficient of inner meshing increase; the load sharing coefficient of outer meshing increases while the maximum meshing force of outer meshing fluctuates near the support stiffness of $10^{7} \mathrm{~N} / \mathrm{m}$. Thirdly, with the increase of support stiffness of ring gear increase, both of the maximum meshing force and load sharing coefficient of inner meshing decrease while these of outer meshing increase. Finally, there is mere change of the maximum meshing force and load sharing coefficient of inner meshing and outer meshing, no matter how much the support stiffness of carrier changes.

\section{Impact of Torsional Stiffness on Dynamic Characteristic of System}

5.1. Impact of Coupling Torsional Stiffness of Gears on the Dynamic Characteristics. Suppose the support stiffness of system is the commendatory value, the torsional stiffness 


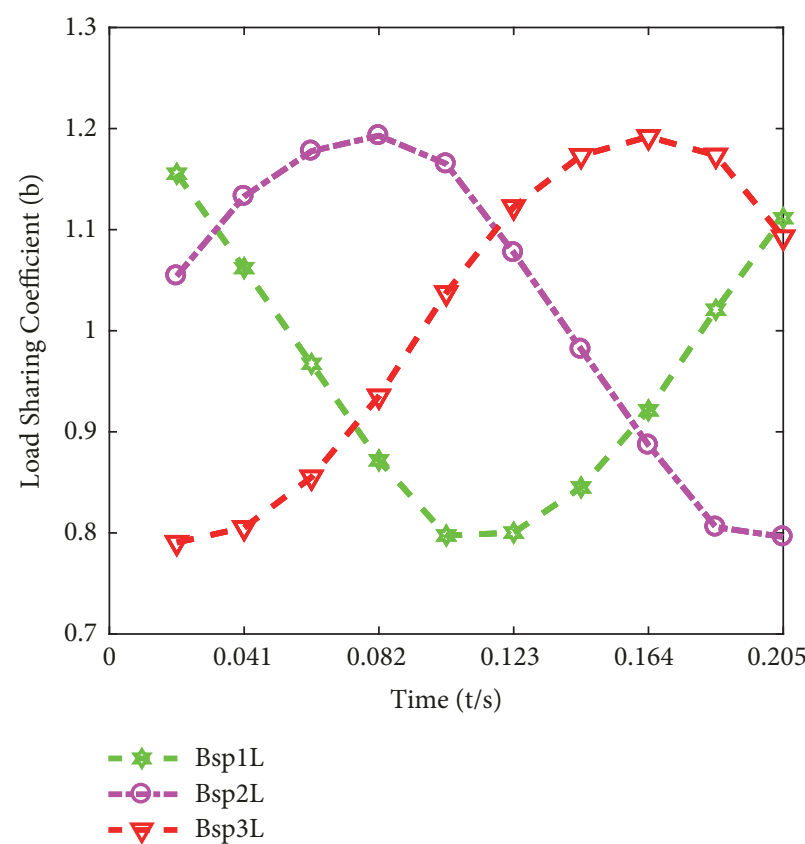

(a) The left-side $L S C$ of inner mesh

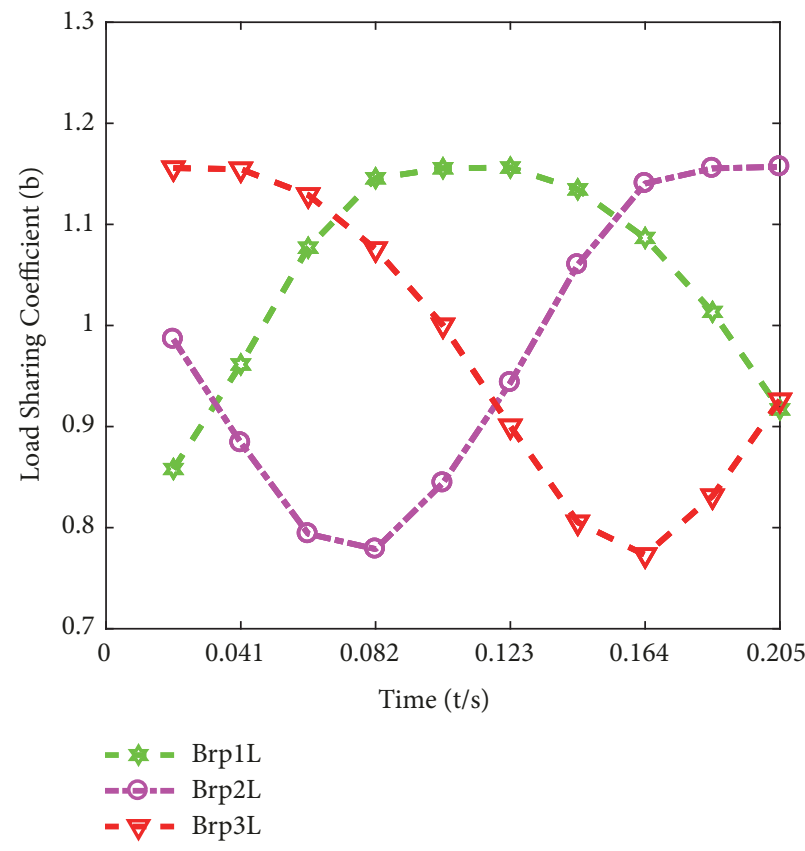

(c) The left-side $L S C$ of outer mesh

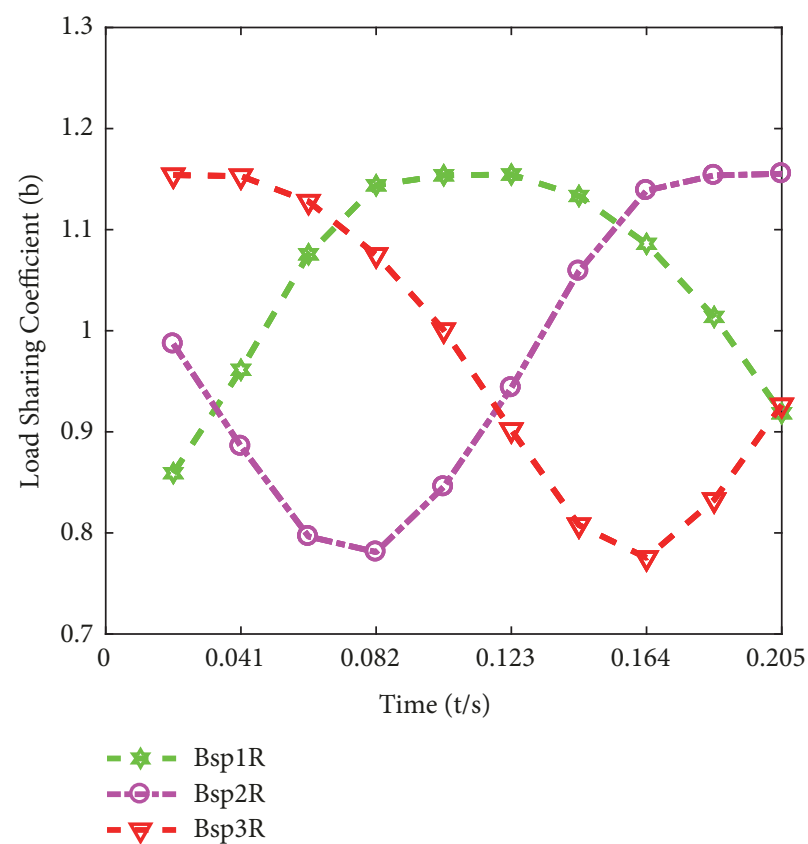

(b) The right-side $L S C$ of inner mesh

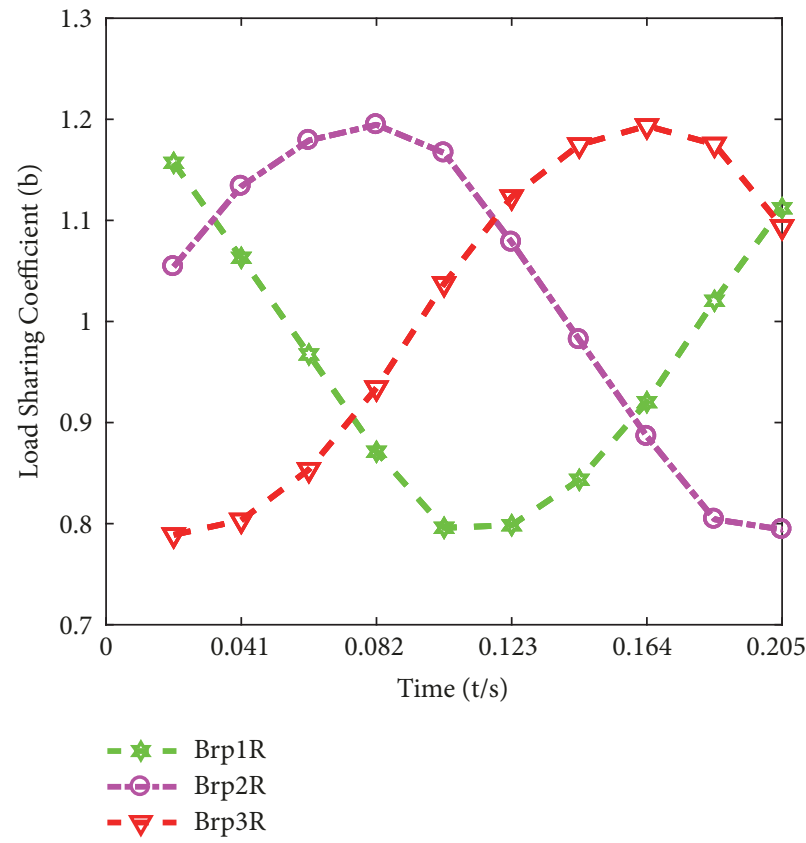

(d) The right-side $L S C$ of outer mesh

FIGURE 24: LSC curves of commendatory parameter.

of left-right helical gear is changed to study the impact of torsional stiffness on dynamic characteristic of system. The torsional stiffness of planetary gear and ring gear is a constant value that is $10^{8} \mathrm{Nm} / \mathrm{rad}$, while changing it of sun gear which is $10^{7}, 5 \times 10^{7}, 10^{8}, 5 \times 10^{8}$, and $10^{9} \mathrm{Nm} / \mathrm{rad}$, respectively. The inner and outer meshing force and load sharing coefficient of system are shown in Figures 25 and 26.
Likewise, when the torsional stiffness of sun gear and ring gear is a constant value, the curves of maximum meshing force and load sharing coefficient with the changing of torsional stiffness of planetary gear from $10^{7} \mathrm{Nm} / \mathrm{rad}$ to $10^{9}$ $\mathrm{Nm} / \mathrm{rad}$ are shown in Figures 27 and 28. Change the torsional stiffness of ring gear; the curves of maximum meshing force and load sharing coefficient with the changing of torsional 


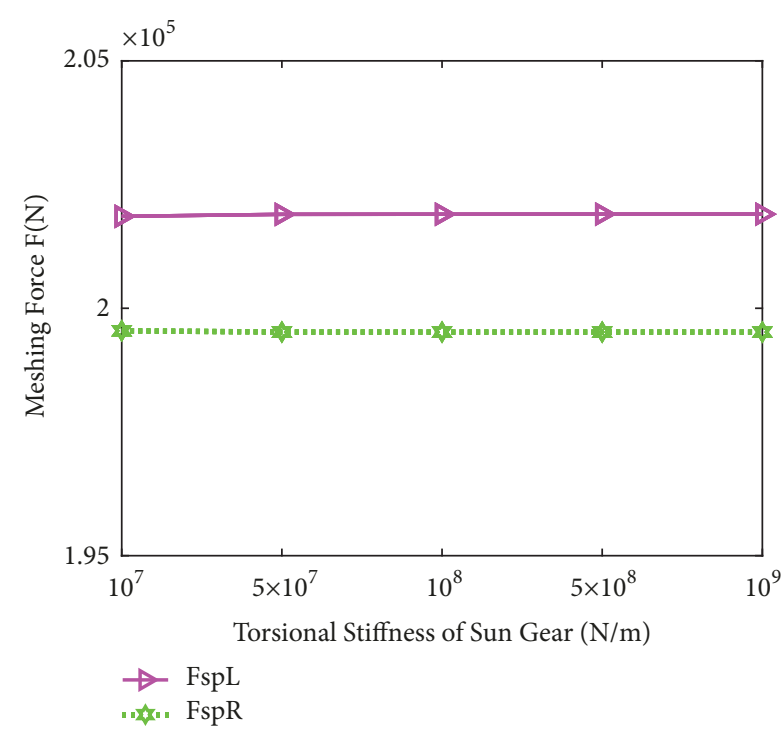

(a) The maximum meshing force of inner meshing

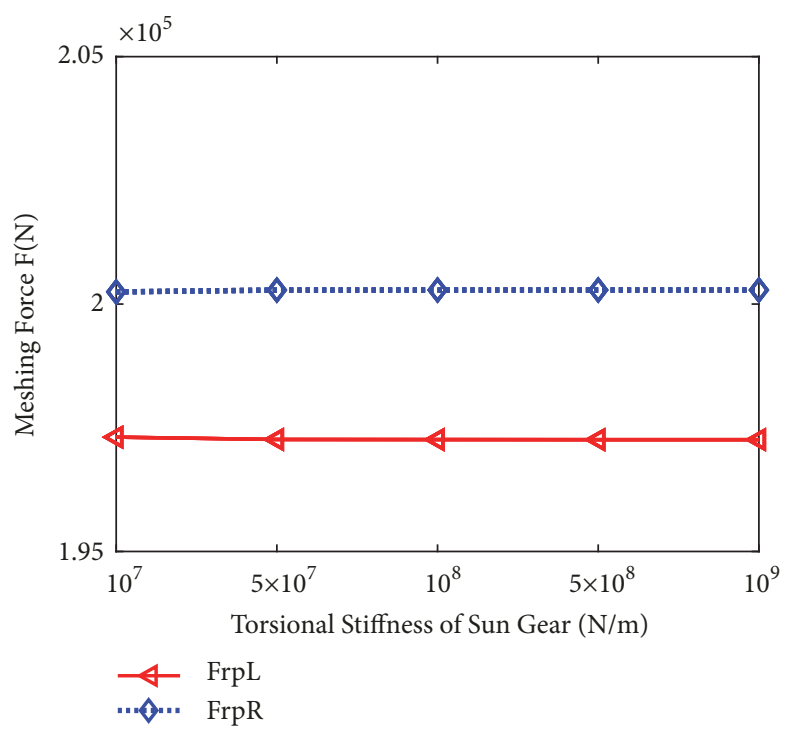

(b) The maximum meshing force of outer meshing

FIGURE 25: Curves of maximum meshing force-torsional stiffness of sun gear.

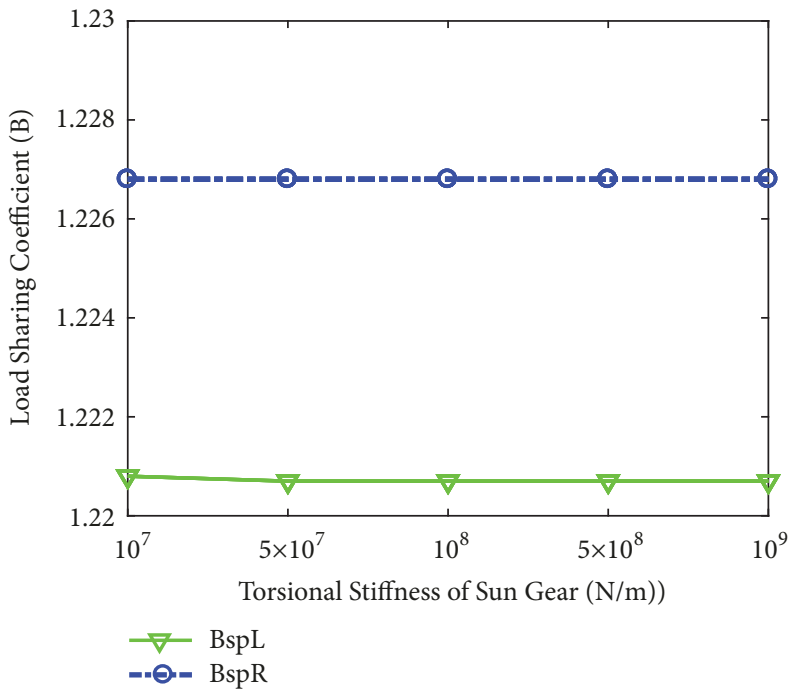

(a) The load sharing coefficient of inner meshing

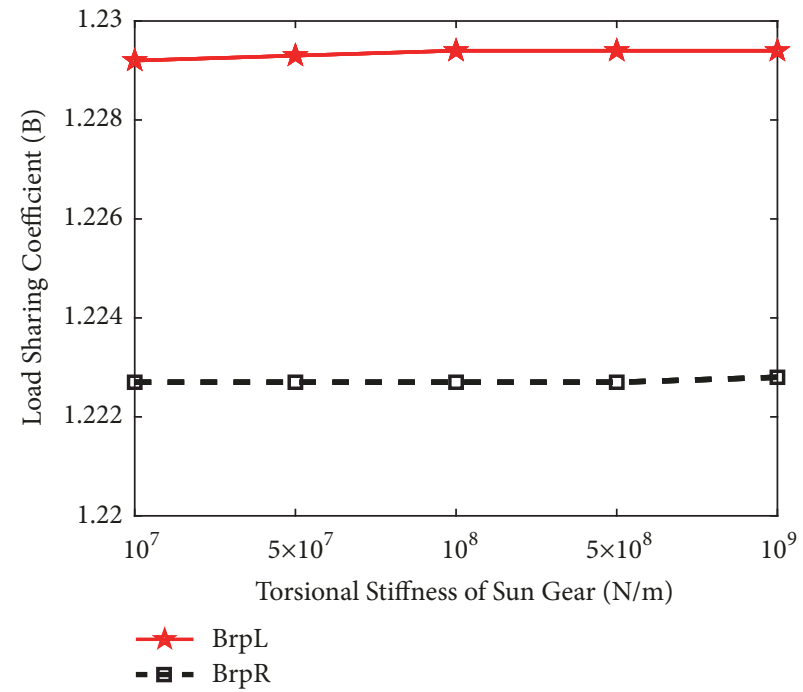

(b) The load sharing coefficient of outer meshing

FIGURE 26: Curves of load sharing coefficient-torsional stiffness of sun gear.

stiffness of ring gear from $10^{7} \mathrm{Nm} / \mathrm{rad}$ to $10^{9} \mathrm{Nm} / \mathrm{rad}$ are shown in Figures 29 and 30.

5.2. Impact of Torsional Stiffness of Carrier on the Dynamic Characteristics. For researching impact of torsional stiffness of carrier on dynamic characteristic of system, the torsional stiffness of carrier is changed from $10^{7} \mathrm{Nm} / \mathrm{rad}$ to $10^{9} \mathrm{Nm} / \mathrm{rad}$, while the left-right coupling torsional stiffness of sun gear, planetary gear, and ring gear is a constant value. The curves of maximum meshing force and load sharing coefficient are shown in Figures 31 and 32.

In summary, the left-right coupling torsional stiffness of sun gear and ring gear has seldom impact on dynamic characteristic when it changes in a reasonable range, and the left-right coupling torsional stiffness of planetary gear has obvious impact on load sharing coefficient but has little impact on maximum meshing force. Likewise, the torsional stiffness of carrier has obvious impact on load sharing coefficient but has little impact on maximum meshing force. 


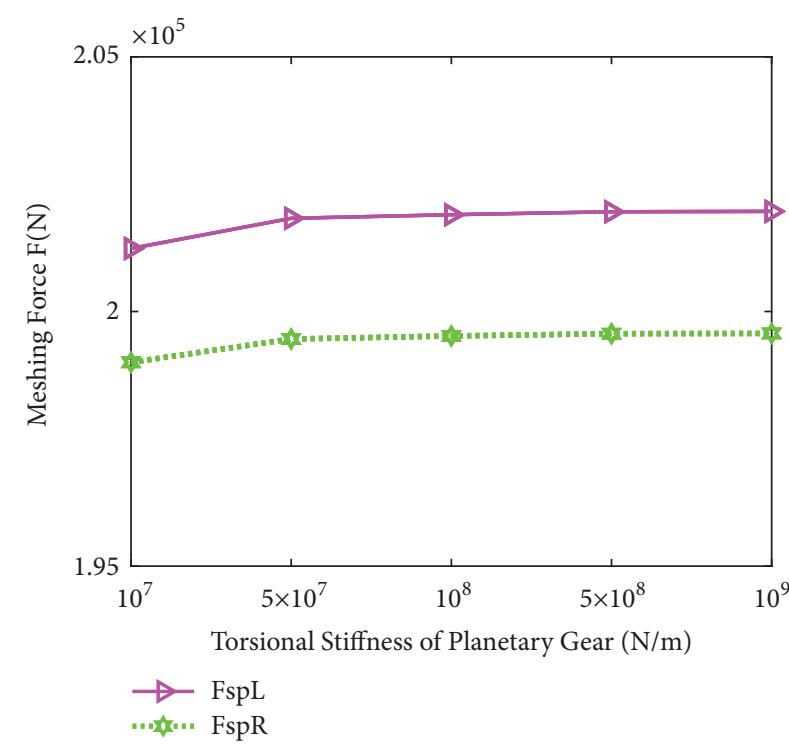

(a) The maximum meshing force of inner meshing

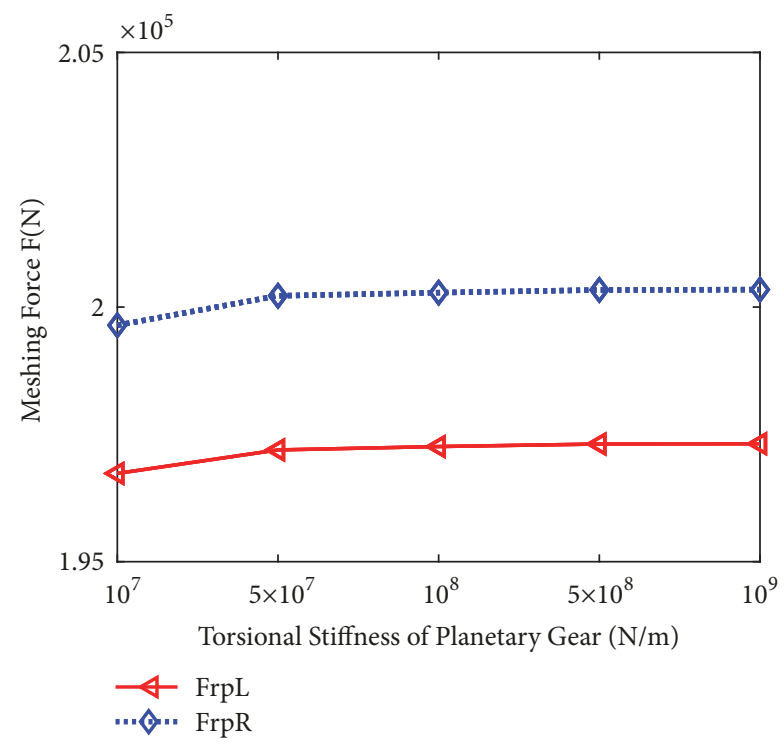

(b) The maximum meshing force of outer meshing

FIGURE 27: Curves of maximum meshing force-torsional stiffness of planetary gear.

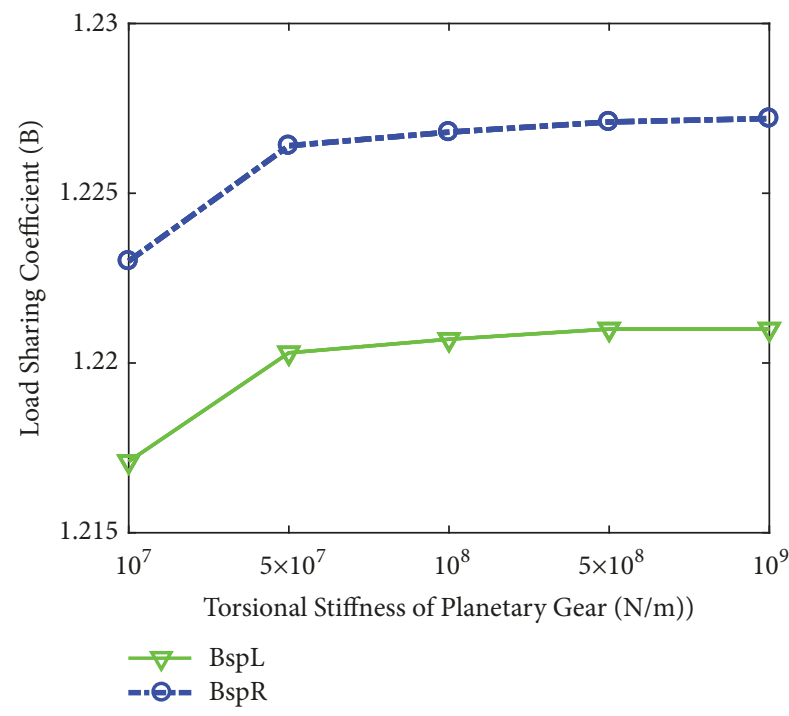

(a) The load sharing coefficient of inner meshing

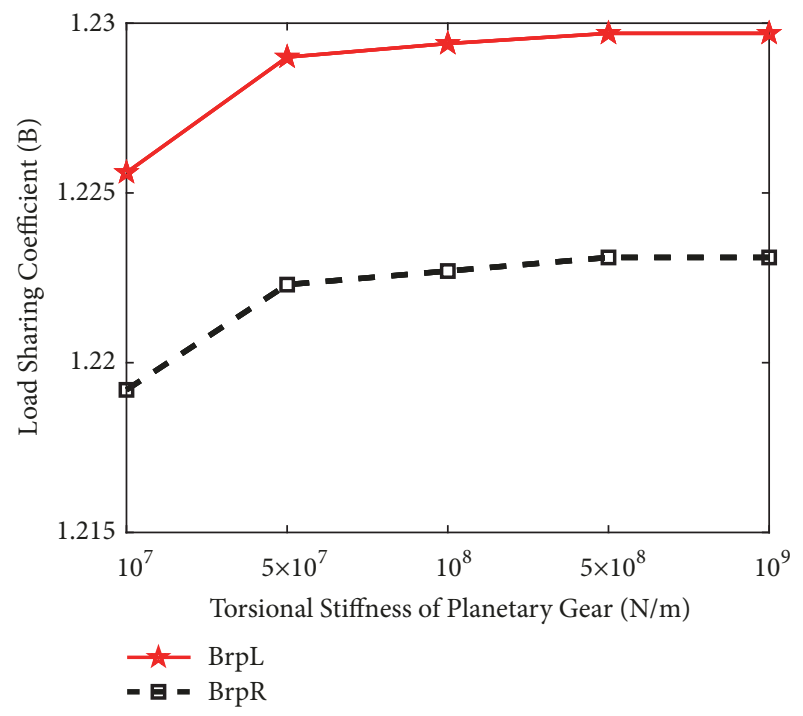

(b) The load sharing coefficient of outer meshing

FIGURE 28: Curves of load sharing coefficient-torsional stiffness of planetary gear.

\section{Dynamic Simulation Verifications}

To verify the validity of theoretical calculation, 3D model of the researched system is built to be simulation analyzed in commercial software ADAMS. The similar simulation curves are fitted together, as shown in Figure 33, where simulation curve of meshing force is written as SCMF.

Meanwhile, according to the simulation meshing force, (23) and (24), load sharing coefficient can be calculated. The curve of load sharing coefficient is shown in Figure 34, where LSC means load sharing coefficient and ROM, RIM, $L I M$, and $L O M$ mean right-side outer meshing, right-side inner meshing, left-side inner meshing, and lest-side outer meshing, respectively.

In summary, the tendency and phase distribution of meshing force of simulation curves are similar to these curves of theoretical calculation. And the curves of load sharing coefficient of simulation are relatively consistent with these curves of theoretical calculation. Both of them verify the correctness of theoretical dynamic analysis method. 


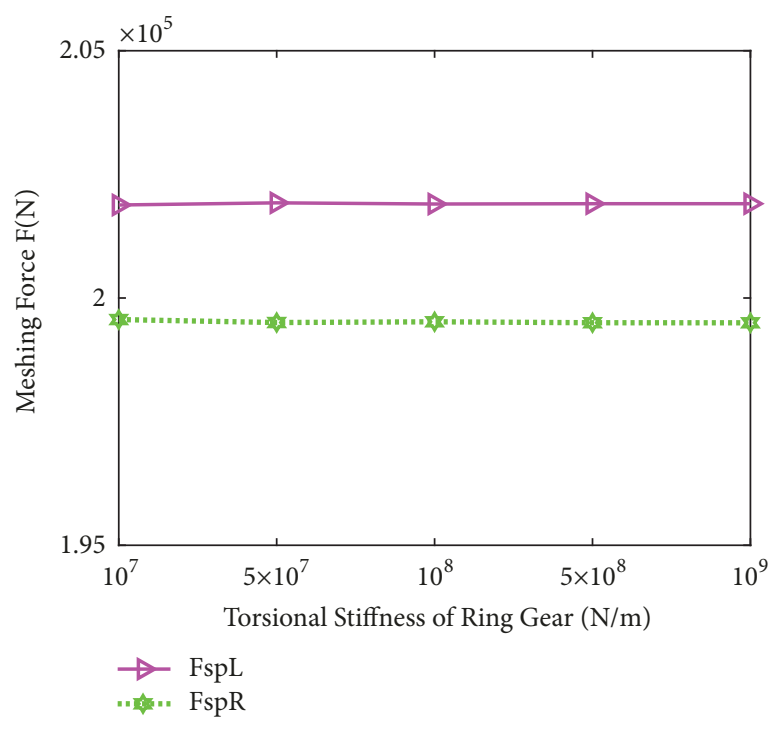

(a) The maximum meshing force of inner meshing

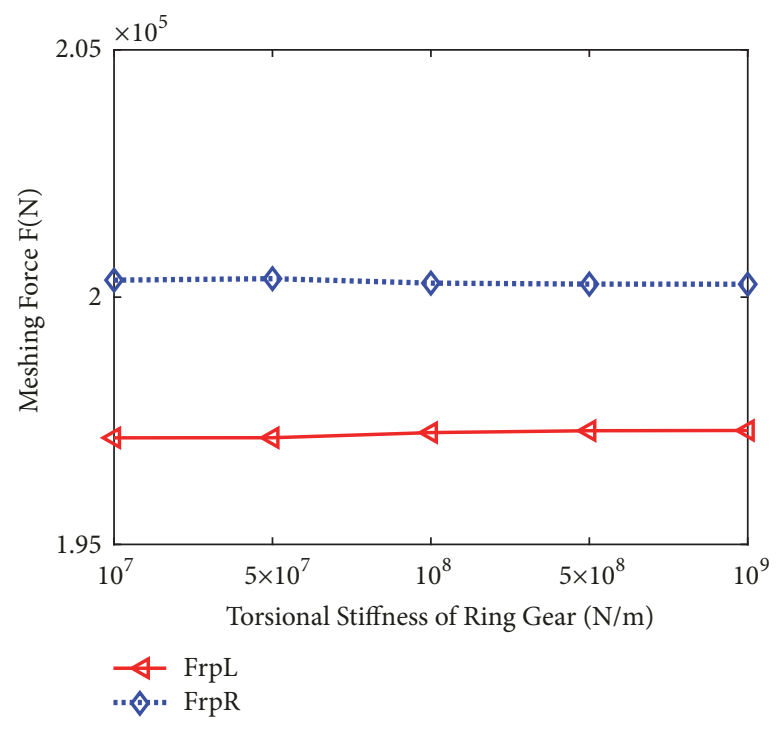

(b) The maximum meshing force of outer meshing

FIGURE 29: Curves of maximum meshing force-torsional stiffness of ring gear.

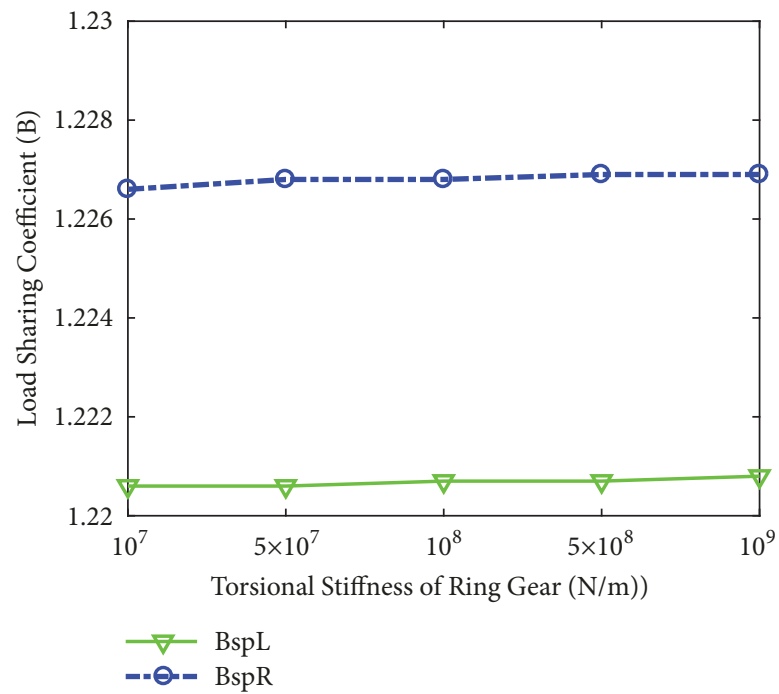

(a) The load sharing coefficient of inner meshing

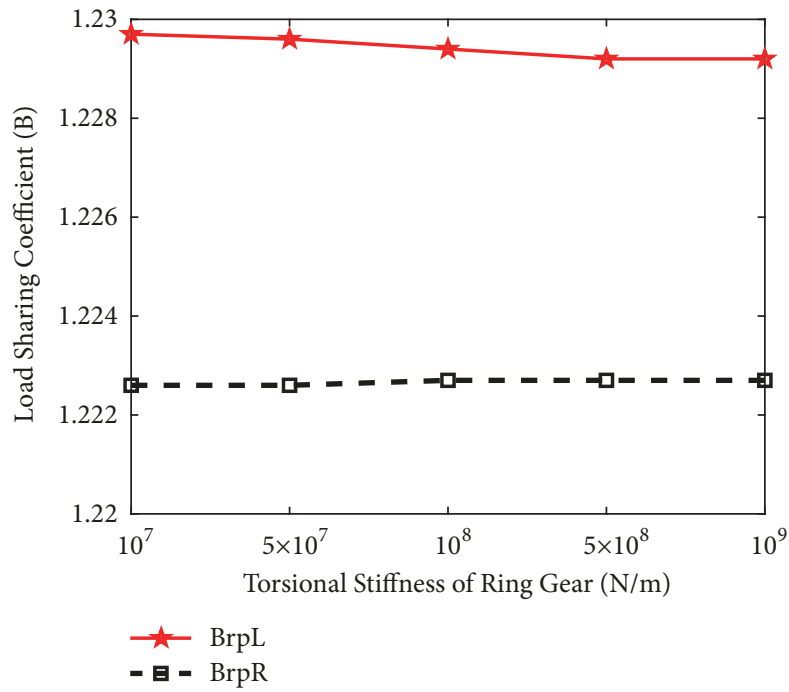

(b) The load sharing coefficient of outer meshing

FIgURE 30: Curves of load sharing coefficient-torsional stiffness of ring gear.

\section{Conclusion}

(1) Considering the impact of support stiffness and leftright coupling torsional stiffness on dynamics characteristic of herringbone gear transmission and the impact of carrier as one part of system, whose dynamic characteristic will influence the system dynamics, the dynamic equations of herringbone gear transmission system were established by lumped parameter method based on system dynamics and Lagrange equation.
(2) Study the impact of stagger angle of gear on dynamic characteristic of system. Stagger angle has obvious impact on load sharing coefficient but little on maximum meshing force. In the processing of stagger angle increase from 0 to $\pi$, both the left and right-side maximum meshing force have an unobvious change. But the load sharing coefficient of left side decreases at first and increases at last, while the right side decreases all the time. The load sharing coefficient of inner meshing meets the minimum when stagger angle is $\pi / 2$, and the outer meshing meets the minimum when stagger 


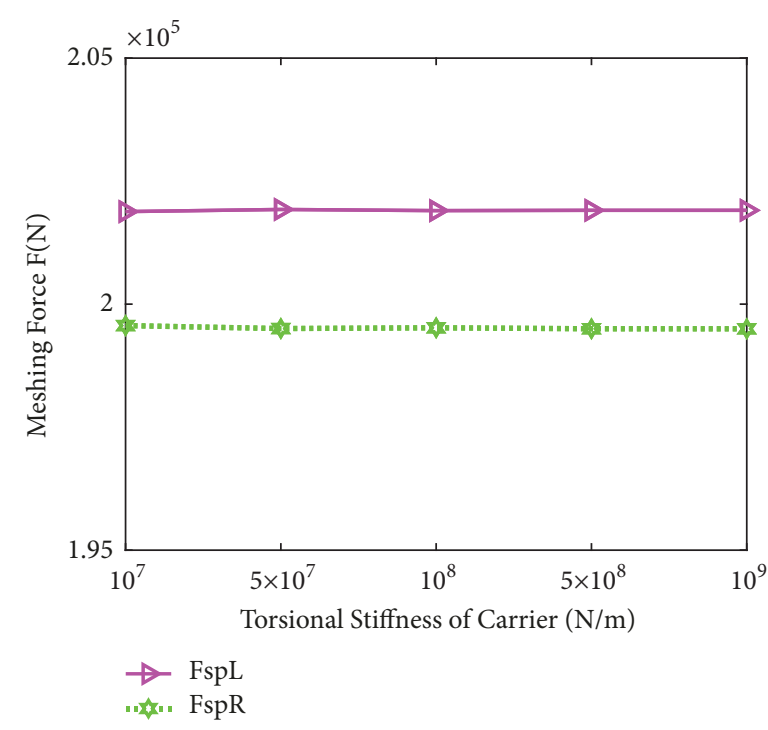

(a) The maximum meshing force of inner meshing

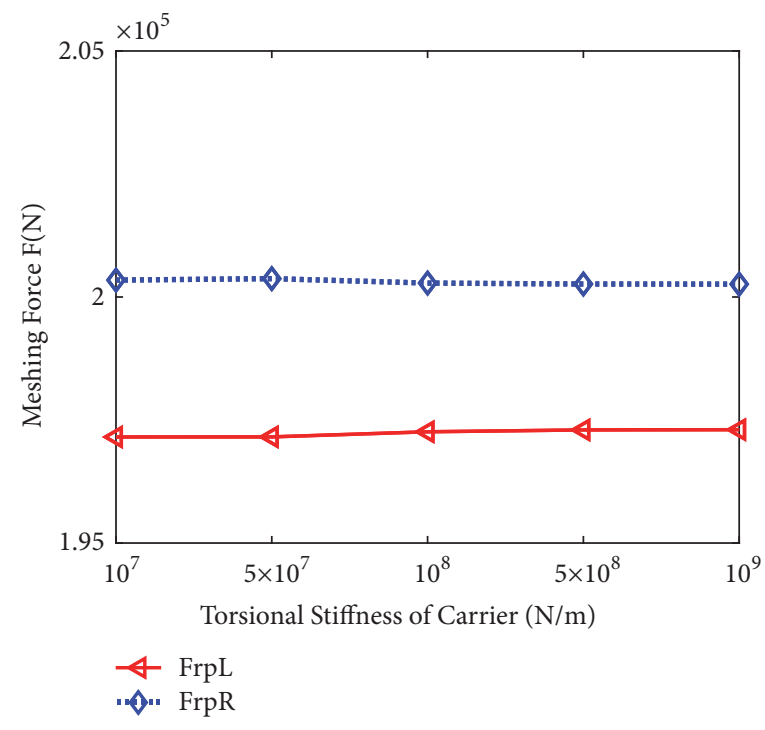

(b) The maximum meshing force of outer meshing

FIGURE 31: Curves of maximum meshing force-torsional stiffness of carrier.

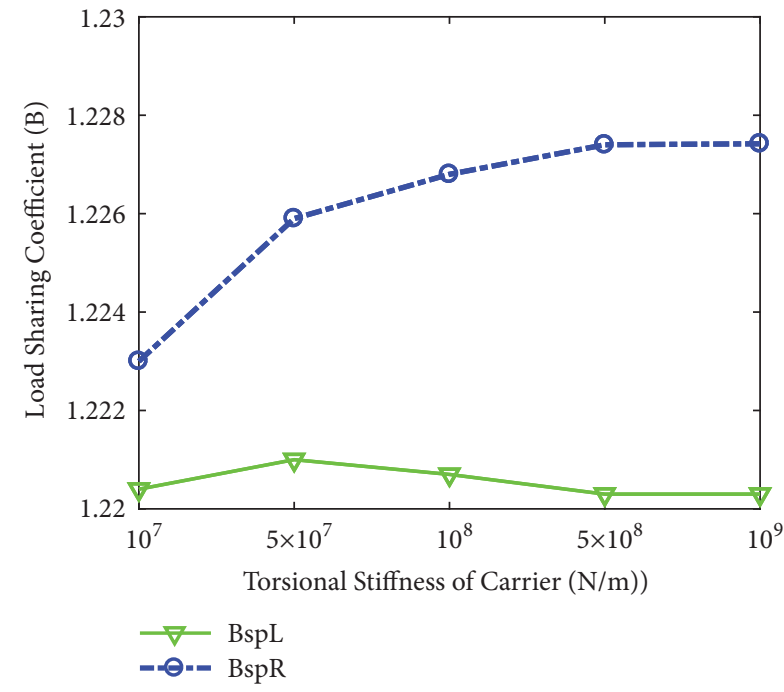

(a) The load sharing coefficient of inner meshing

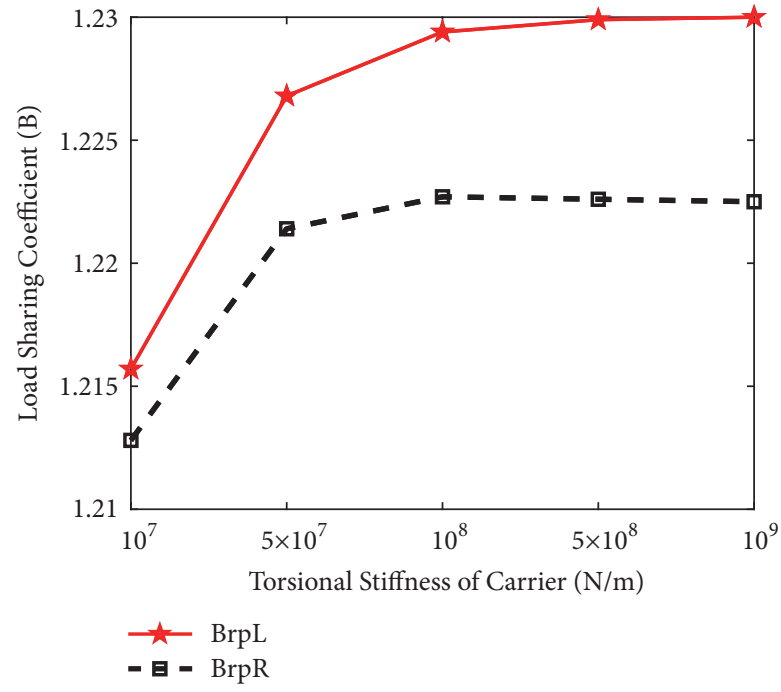

(b) The load sharing coefficient of outer meshing

FIGURE 32: Curves of load sharing coefficient-torsional stiffness of carrier.

angle is $3 \pi / 4$, and the best stagger angle of this system is $3 \pi / 4$.

(3) In the herringbone gear transmission system, the changing of support stiffness will have impact on dynamic characteristics. With the increase of the support stiffness of sun gear, both of the maximum meshing force and load sharing coefficient of inner meshing increase while these of outer meshing decrease. With the increase of support stiffness of planetary gear, both of the maximum meshing force and load sharing coefficient of inner meshing increase; the load sharing coefficient of outer meshing increases while the maximum meshing force of outer meshing fluctuates near the support stiffness of $10^{7} \mathrm{~N} / \mathrm{m}$. With the increase of support stiffness of ring gear increase, both of the maximum meshing force and load sharing coefficient of inner meshing decrease while these of outer meshing increase. And, there is mere change of the maximum meshing force and load sharing coefficient of inner meshing and outer meshing, no matter how much the support stiffness of carrier changes. The commendatory support stiffness of this paper is as follows: all of support stiffness of sun gear, planetary gear, ring gear, and carrier is $10^{7} \mathrm{~N} / \mathrm{m}$.

(4) The impact of torsional stiffness on system characteristic was considered. The meshing force and load sharing coefficient seldomly changed while the changing of left-right coupling torsional stiffness changed from $10^{7} \mathrm{~N} \mathrm{~m} / \mathrm{rad}$ to 


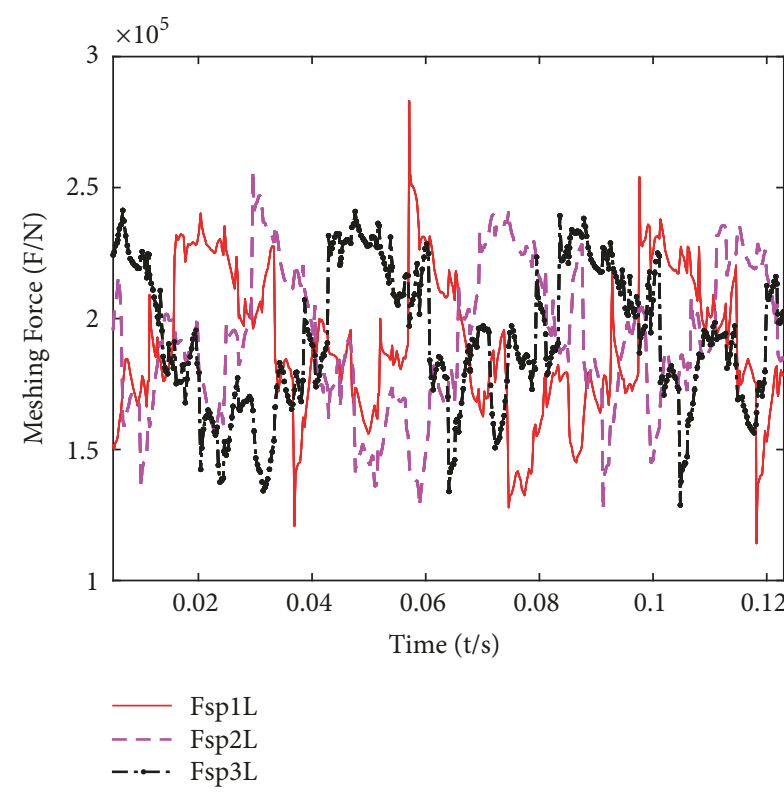

(a) SCMF of inner meshing of left-side

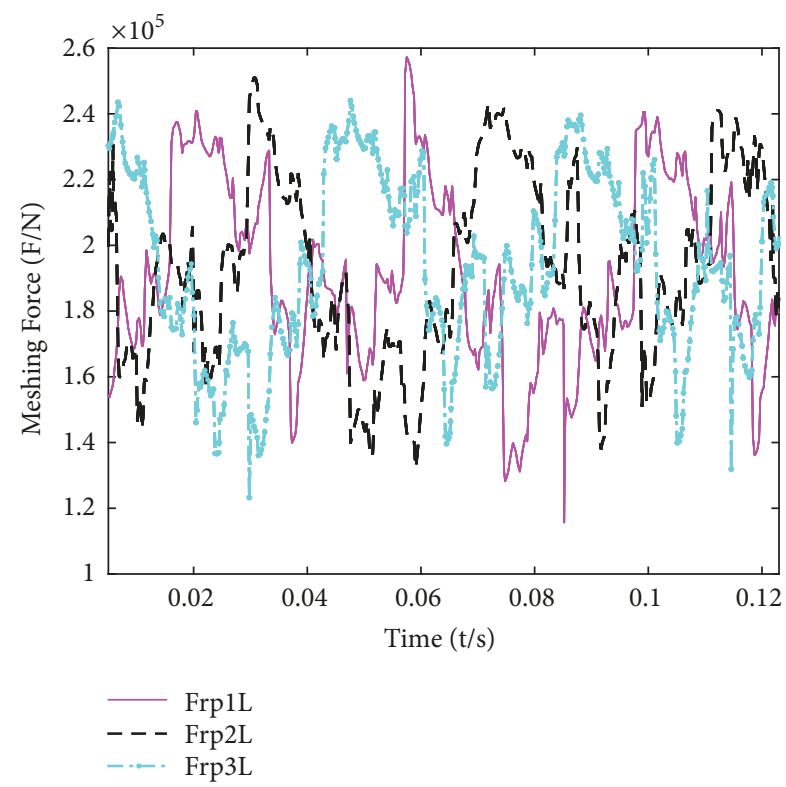

(c) SCMF of outer meshing of left-side

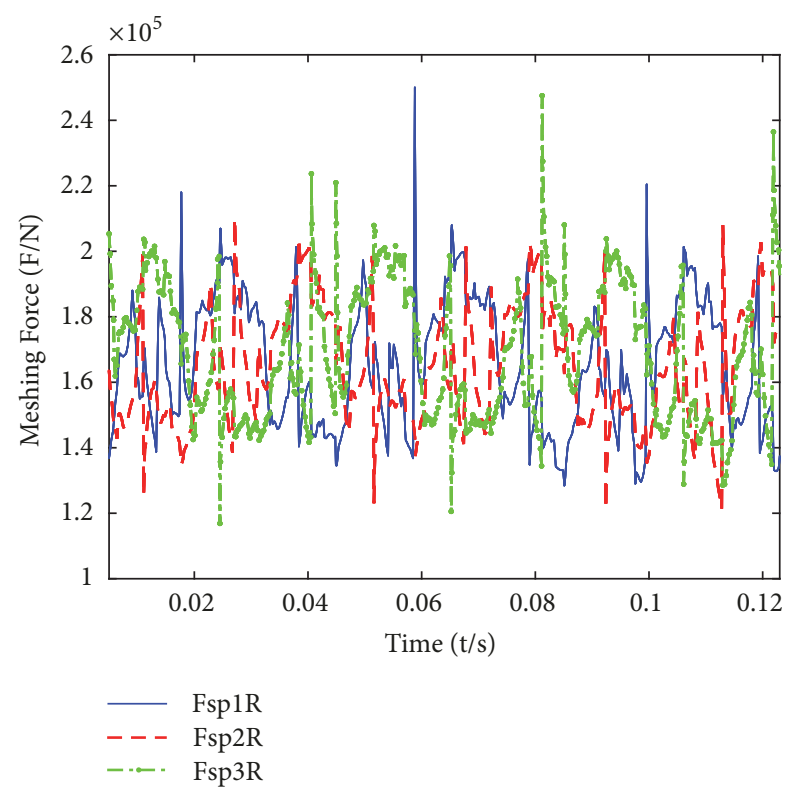

(b) SCMF of inner meshing of right-side

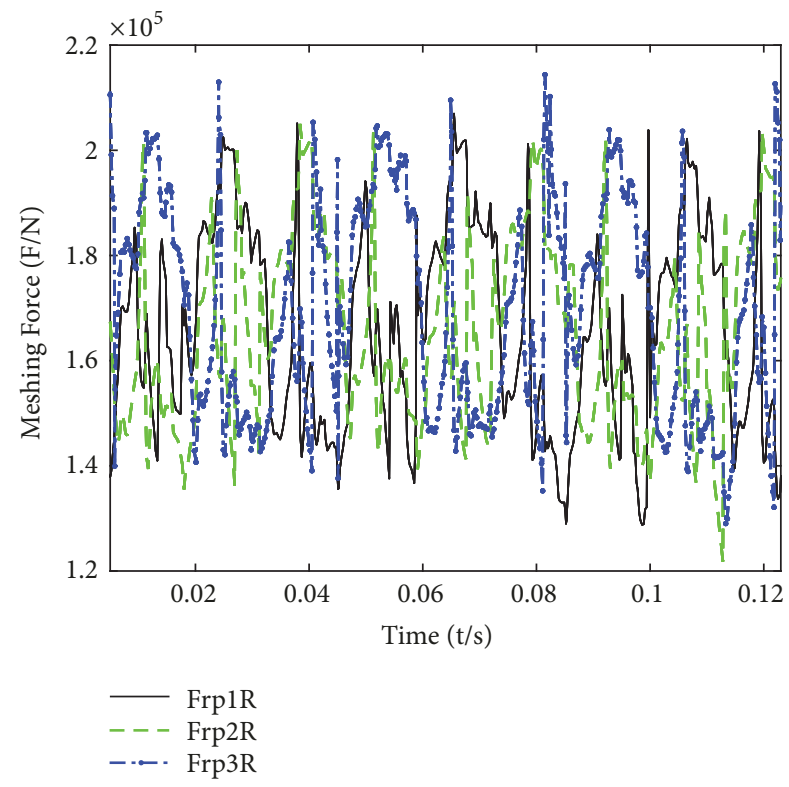

(d) SCMF of outer meshing of right-side

FIGURE 33: Simulation curves of meshing force of researched system.

$10^{9} \mathrm{Nm} / \mathrm{rad}$ except the change of coupling torsional stiffness of planetary gear. When the coupling torsional stiffness changed from $10^{7} \mathrm{~N} \mathrm{~m} / \mathrm{rad}$ to $10^{9} \mathrm{Nm} / \mathrm{rad}$, the maximum meshing force is nearly a constant value while the load sharing coefficient increases slowly. Moreover, with the change of torsional stiffness of carrier, the meshing force is merely changed and the load sharing coefficient changes a lot.

(5) The dynamic characteristic was simulated by commercial software ADAMS. And curves of meshing force and load sharing coefficient gotten from ADAMS simulation were compared with these of theoretical calculation. The result is shown that both the meshing force curves and the load sharing curves are in good agreement with the theoretical curves, which validates the necessity and correctness of theoretical analysis method.

\section{Data Availability}

All data generated or analyzed during this study are included in this published article, and other pieces of information are available from the corresponding author on reasonable request. 


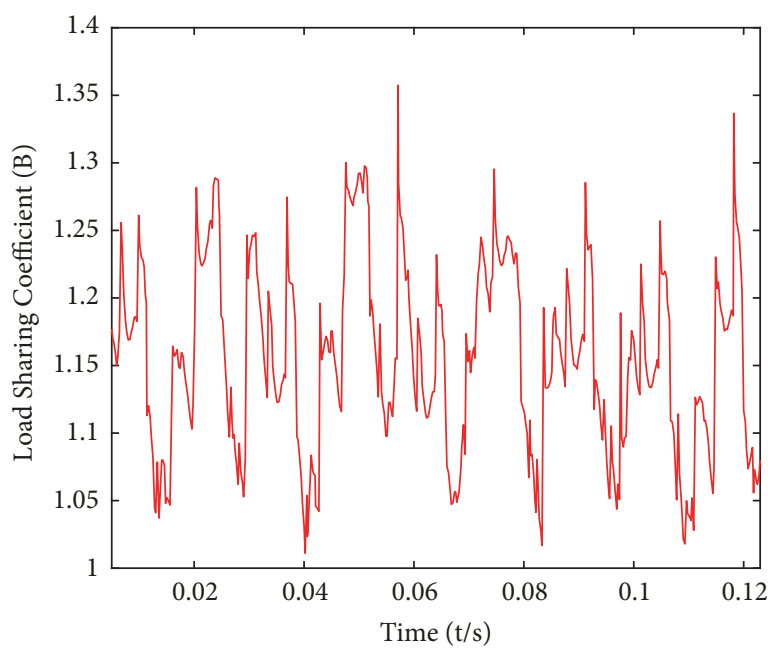

— The Maximum LSC of LIM

(a) The Maximum LSC of LIM

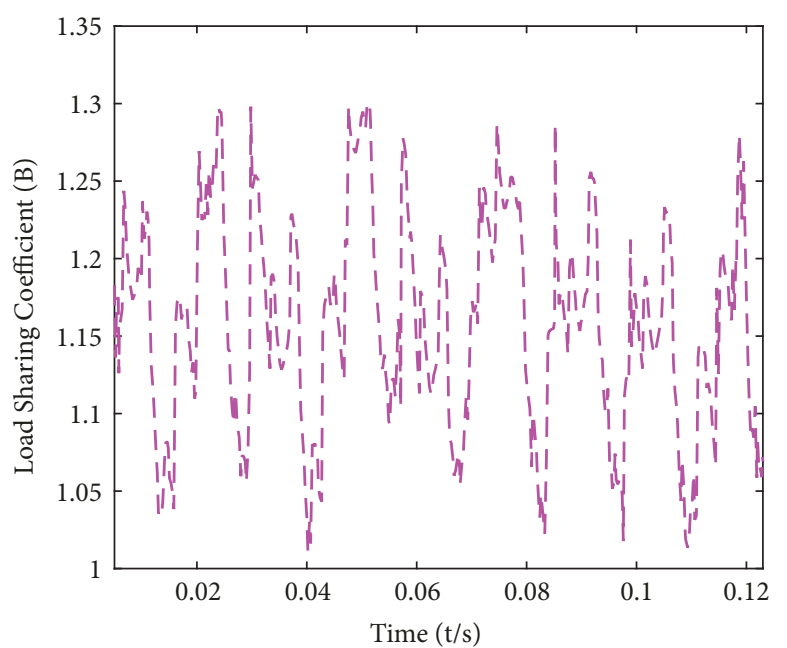

- - - The Maximum LSC of LOM

(c) The Maximum LSC of LOM

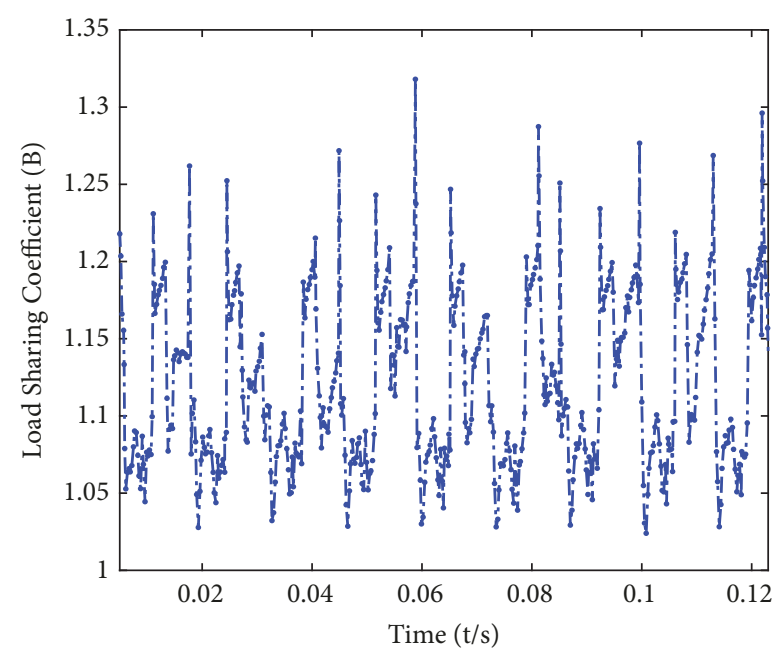

-...-. The Maximum LSC of RIM

(b) The Maximum LSC of RIM

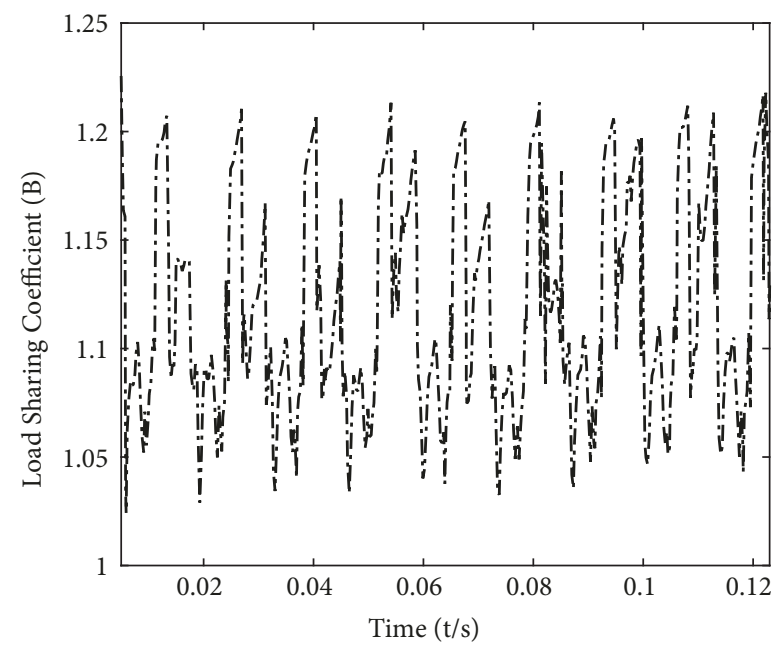

-.-. The Maximum LSC of ROM

(d) The Maximum LSC of ROM

Figure 34: Curves of load sharing coefficient by simulation.

TABLE 1: The wind power gearbox parameters.

\begin{tabular}{lcccc}
\hline Parameters & Unit & Sun gear & Planetary gear & Ring gear \\
Number of teeth & $/$ & 22 & 41 & 104 \\
Modulus & $\mathrm{mm}$ & 16 & 16 & 16 \\
Mass & $\mathrm{kg}$ & 149.642 & 517.8 & 671.288 \\
Pressure angle & $\mathrm{deg}$ & 20 & 20 & 20 \\
Helix angle & $\mathrm{deg}$ & 15 & 15 & 15 \\
Mesh stiffness & $\mathrm{N} / \mathrm{m}$ & $2.536 \times 10^{10}$ & & $3.608 \times 10^{10}$ \\
Mesh damping & $\mathrm{N} / \mathrm{m} \cdot \mathrm{kg}$ & $2 \xi\left(\frac{K_{s p}}{1 / \mathrm{m}_{\mathrm{s}}+1 / \mathrm{m}_{\mathrm{p}}}\right)^{1 / 2}$ & & $K_{r p}$ \\
Input speed & $\mathrm{r} / \mathrm{min}$ & $\mathrm{kW}$ & & $2 \xi\left(\frac{1 / \mathrm{m}_{\mathrm{r}}+1 / \mathrm{m}_{\mathrm{p}}}{1 / 2}\right.$ \\
Input power & & & 14.18 \\
\hline
\end{tabular}

Note. $\xi$ is an arbitrary value in the range of $0.07-0.13$. $\xi$ is 0.1 in this paper. 
TABLE 2: The initial amplitude and phase of transmission error.

\begin{tabular}{lcccc}
\hline Harmonic number L & $\widehat{e}_{\text {spl }} / \mu \mathrm{m}$ & $\sigma_{\text {spl }} / \mathrm{rad}$ & $\widehat{e}_{r p l} / \mu \mathrm{m}$ & $\sigma_{r p l} / \mathrm{rad}$ \\
\hline 1 & 2.315 & -0.658 & 2.131 & -0.835 \\
2 & 0.221 & -1.346 & 0.115 & -1.389 \\
3 & 0.082 & 0.047 & 0.051 & 0.983 \\
\hline
\end{tabular}

\section{Conflicts of Interest}

The authors declare that they have no conflicts of interest.

\section{Acknowledgments}

This research is supported by National Natural Science Foundation of China (no. 51805368), Natural Science Foundation of Tianjin (no. 17JCQNJC04300), Open Funding of the State Key Laboratory of Materials Processing and Die \& Mould Technology-Huazhong University of Science and Technology (no. P2019-022), Fundamental Research Funds for the Tianjin Universities (no. 2017KJ083), Applied Basic Research Project of China Textile Industry Association (no. J201806), and the Program for Innovative Research Team in University of Tianjin (no. TD13-5037). Thanks also go to Professor Haruo Houjoh, Shigeki Matsumura of Tokyo Institute of Technology, Professor Izhak Bucher of Israel Institute of Technology, and Professor Yidu Zhang of Beihang University for their constant assistances.

\section{References}

[1] W. Shi, H. Qiu, Z. Han, and P. Qin, "Dynamics research of wind turbine gearbox with herringbone planetary gear," Chongqing Daxue Xuebao/Journal of Chongqing University, vol. 38, no. 1, pp. 95-132, 2015.

[2] L. Zhang, "Impact of Meshing Phase on Load Sharing for Herringbone Planetary Train," Journal of Mechanical Engineering, vol. 54, no. 11, pp. 129-140, 2018.

[3] S. Mo, Y. Zhang, Q. Wu, F. Wang, S. Matsumura, and H. Houjoh, "Load Sharing Behavior of Star Gearing Reducer for Geared Turbofan Engine," Chinese Journal of Mechanical Engineering, vol. 30, no. 4, pp. 796-803, 2017.

[4] S. Mo, Y. Zhang, Q. Wu, H. Houjoh, and S. Matsumura, "Research on natural characteristics of double-helical star gearing system for GTF aero-engine," Mechanism and Machine Theory, vol. 106, pp. 166-189, 2016.

[5] S. Mo, Y. Zhang, and Q. Wu, "Research on multiple-split load sharing of two-stage star gearing system in consideration of displacement compatibility," Mechanism and Machine Theory, vol. 88, pp. 1-15, 2015.

[6] M. Li, L. Xie, and L. Ding, "Load sharing analysis and reliability prediction for planetary gear train of helicopter," Mechanism and Machine Theory, vol. 115, pp. 97-113, 2017.

[7] Z. Zhu, R. Zhu, Y. Li, Y. Chen, and Z. Zhu, "Impact of installation error on dynamics load sharing characteristic for encased differential herringbone train," Jixie Gongcheng Xuebao/Journal of Mechanical Engineering, vol. 48, no. 3, pp. 16-24, 2012.

[8] J.-H. Lu, R.-P. Zhu, and G.-H. Jin, "Basic-components' offset analysis of planetary gearing in need of loading sharing," Zhongnan Daxue Xuebao (Ziran Kexue Ban)/Journal of Central
South University (Science and Technology), vol. 39, no. 1, pp. 143148, 2008.

[9] C. Zhu, X. Xu, T. C. Lim, X. Du, and M. Liu, "Effect of flexible pin on the dynamic behaviors of wind turbine planetary gear drives," Proceedings of the Institution of Mechanical Engineers, Part C: Journal of Mechanical Engineering Science, vol. 227, no. 1, pp. 74-86, 2013.

[10] A. Al-Shyyab and A. Kahraman, "Non-linear dynamic analysis of a multi-mesh gear train using multi-term harmonic balance method: period-one motions," Journal of Sound and Vibration, vol. 284, no. 1-2, pp. 151-172, 2005.

[11] M. Inalpolat, M. Handschuh, and A. Kahraman, "Influence of indexing errors on dynamic response of spur gear pairs," Mechanical Systems and Signal Processing, vol. 60, pp. 391-405, 2015.

[12] A. Bodas and A. Kahraman, "Influence of carrier and gear manufacturing errors on the static load sharing behavior of planetary gear sets," JSME International Journal Series $C$ Mechanical Systems, Machine Elements and Manufacturing, vol. 47, no. 3, pp. 908-915, 2004.

[13] H. Ligata, A. Kahraman, and A. Singh, "An experimental study of the influence of manufacturing errors on the planetary gear stresses and planet load sharing," Journal of Mechanical Design, vol. 130, no. 4, Article ID 041701, 2008.

[14] A. Kahraman, "Load sharing characteristics of planetary transmissions," Mechanism and Machine Theory, vol. 29, no. 8, pp. 1151-1165, 1994.

[15] S. Xue and I. Howard, "Dynamic modelling of flexibly supported gears using iterative convergence of tooth mesh stiffness," Mechanical Systems and Signal Processing, vol. 80, pp. 460-481, 2016.

[16] J. Wang, G. He, J. Zhang, Y. Zhao, and Y. Yao, "Nonlinear dynamics analysis of the spur gear system for railway locomotive," Mechanical Systems and Signal Processing, vol. 85, pp. 4155, 2017.

[17] A. Fernandez-del-Rincon, P. Garcia, A. Diez-Ibarbia, A. deJuan, M. Iglesias, and F. Viadero, "Enhanced model of gear transmission dynamics for condition monitoring applications: Effects of torque, friction and bearing clearance," Mechanical Systems and Signal Processing, vol. 85, pp. 445-467, 2017.

[18] X. Liang, M. J. Zuo, and Z. Feng, "Dynamic modeling of gearbox faults: A review," Mechanical Systems and Signal Processing, vol. 98, pp. 852-876, 2018.

[19] Y. Tan, C. Hu, Y. Zhang, W. Wang, S. Xiong, and H. Zuo, “Testing research on dynamic load sharing performance of encased differential planetary gearbox," Jixie Gongcheng Xuebao/Journal of Mechanical Engineering, vol. 52, no. 9, pp. 28-35, 2016.

[20] A. Singh, "Load sharing behavior in epicyclic gears: physical explanation and generalized formulation," Mechanism and Machine Theory, vol. 45, no. 3, pp. 511-530, 2010.

[21] C. H. Wink and A. L. Serpa, "Performance assessment of solution methods for load distribution problem of gear teeth," Mechanism and Machine Theory, vol. 43, no. 1, pp. 80-94, 2008. 


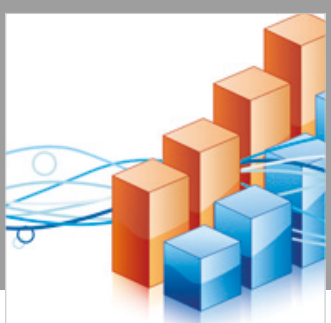

Advances in

Operations Research

\section{-n-m}
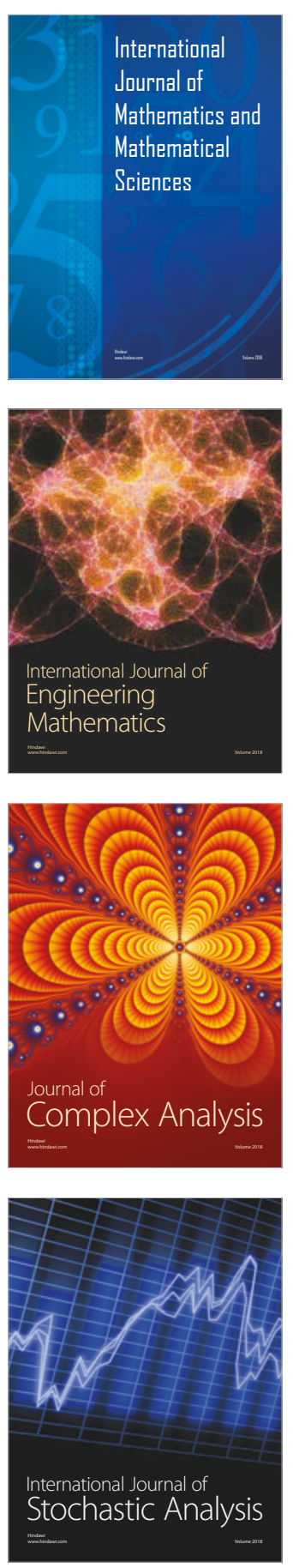
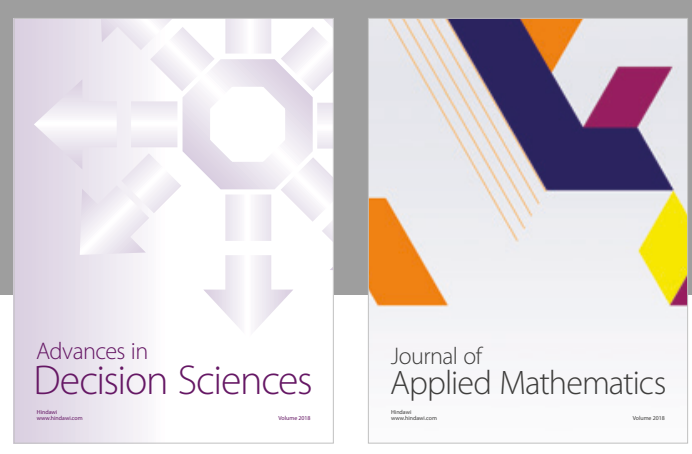

Journal of

Applied Mathematics
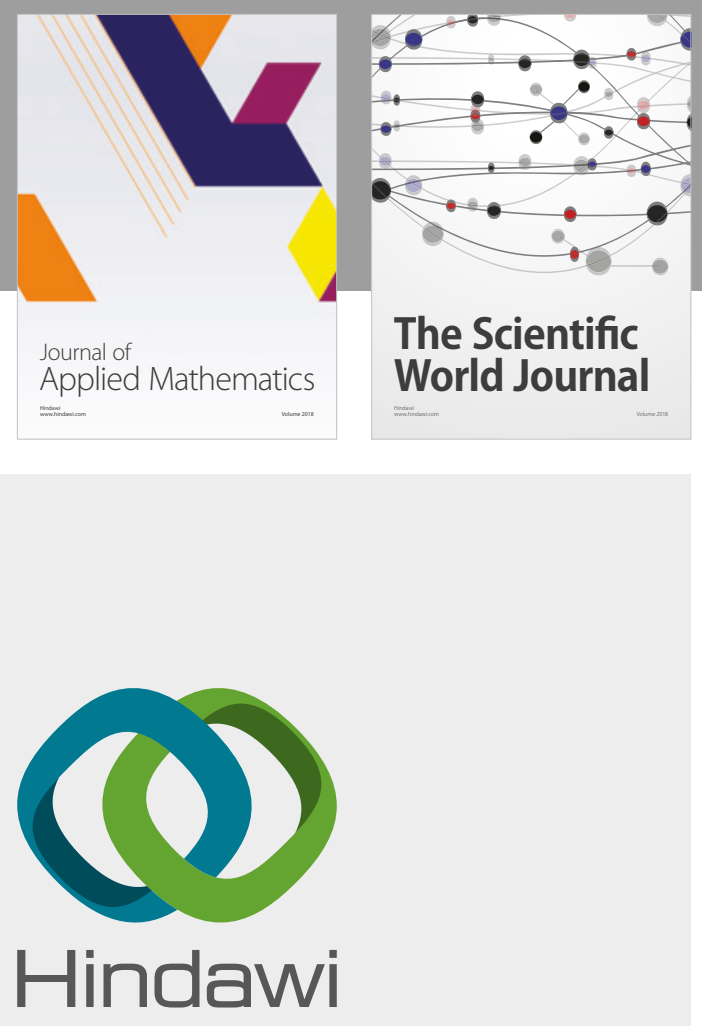

Submit your manuscripts at

www.hindawi.com

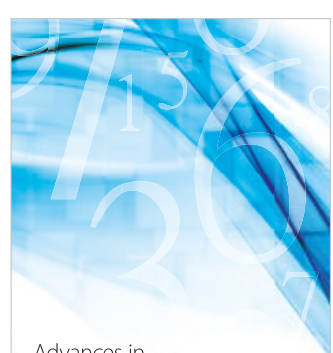

Advances in
Numerical Analysis
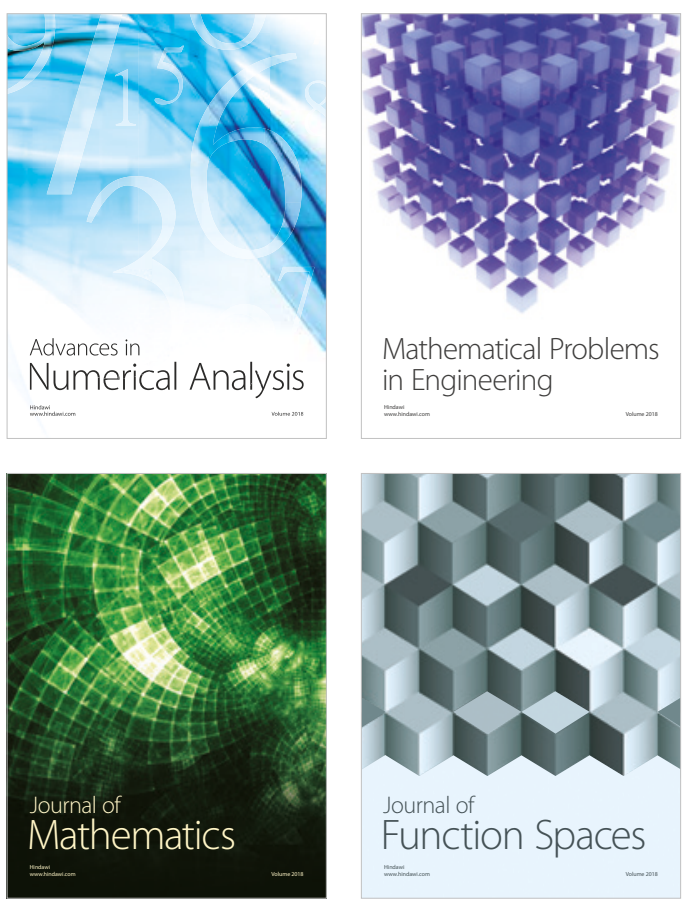

Mathematical Problems in Engineering

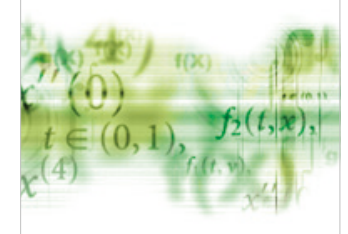

International Journal of

Differential Equations

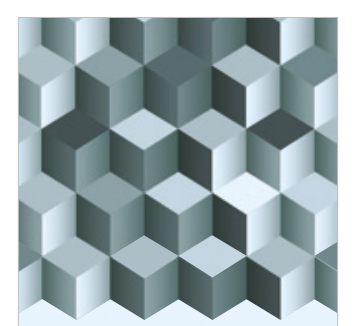

Journal of

Function Spaces

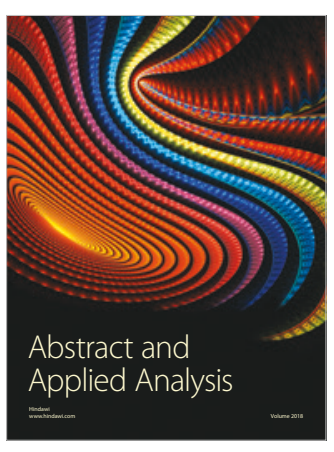

The Scientific

World Journal

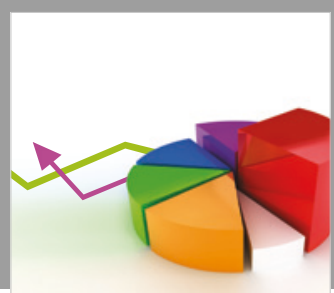

Journal of

Probability and Statistics
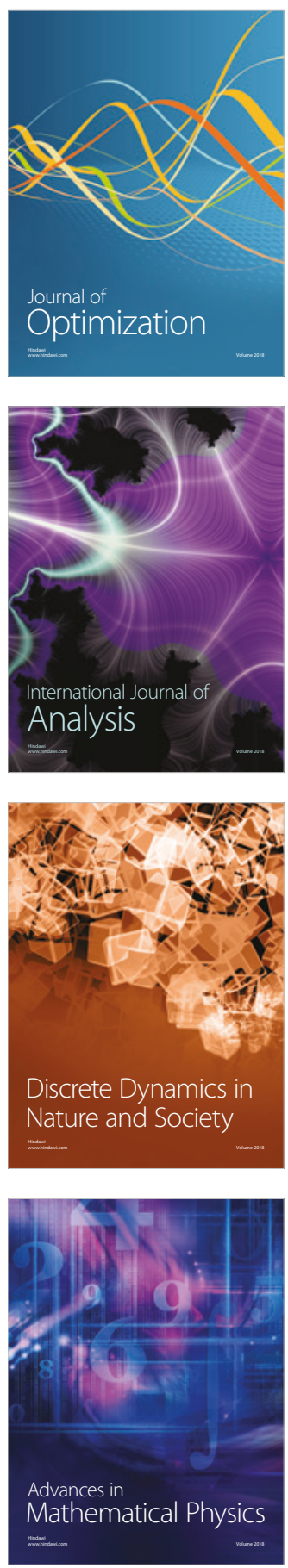